\title{
Revisión bibliográfica de la aplicación de la metodología DEA en Colombia por actividad económica ${ }^{1}$
}

\author{
Bibliographic review of the application of the \\ DEA methodology in Colombia to economic activity
}

Recibido: 16 de marzo de 2016 - Revisado: 13 de junio de 2016 - Aceptado: 27 de junio de 2016

Julia Andrea Pineda Acero ${ }^{2}$

Arcadio Cervera Muñoz ${ }^{3}$

Wilson Oviedo García ${ }^{4}$

\section{Resumen}

Este artículo presenta los resultados de una revisión bibliográfica de la producción intelectual nacional asociada a la aplicación de la metodología de Análisis Envolvente de Datos (DEA, por su sigla en inglés) en Colombia y a la Clasificación Industrial Internacional Uniforme (CIIU) adaptada para el país. A partir de dicha revisión, se establecen las características de los objetos de estudio de las investigaciones y se presenta cómo se usa la metodología. Al concluir, se tiene que, aunque se ha incrementado el manejo de DEA, aún es incipiente $\mathrm{y}$, por ende, existen varias oportunidades de investigación que incluyen diferentes tipos de unidades de decisión (DMU).

\section{Palabras clave}

Análisis Envolvente de Datos, DEA, CIIU, eficiencia relativa.

\begin{abstract}
This article presents the results of a bibliographic review of the national intellectual production associated to the application of the Data Envelopment Analysis (DEA) methodology in Colombia and the International Standard Industrial Classification (ISIC) adapted for the country. From this review, the characteristics of the objects of study of the investigations are established and it is shown how the methodology is used. In conclusion, it is necessary that, although the management of DEA has been increased, it is still incipient and, therefore, there are several research opportunities that include different types of Decision Making Units (DMU).
\end{abstract}

\section{Keywords}

Data Envelopment Analysis, DEA, CIIU, relative efficiency.

\footnotetext{
${ }^{1}$ Este artículo es resultado de la investigación titulada: "Aplicación de la metodología DEA para la medición de eficiencias técnicas relativas en sistemas organizacionales", financiada por la Universidad de La Salle, Bogotá, Colombia.

2 Magíster en Administración Universidad de los Andes. Magíster en Ingeniería de sistemas y computación Universidad de los Andes. Ingeniera de diseño y automatización electrónica Universidad de La Salle. Profesora Universidad de La Sabana, Bogotá, Colombia.

Correo electrónico: juliapa@unisabana.edu.co

3 Magíster en Ingeniería industrial Universidad de los Andes. Ingeniero industrial Universidad de América. Profesor Universidad de La Salle, Bogotá, Colombia. Correo electrónico: arcervera@unisalle.edu.co

${ }^{4}$ Magíster en Administración Universidad Nacional de Colombia. Administrador de empresas Universidad del Tolima. Profesor Universidad de la Salle, Bogotá, Colombia.

Correo electrónico: woviedo@unisalle.edu.co

Para citar este artículo use: Pineda, J., Cervera, A., \& García, O (2017). Revisión bibliográfica de la aplicación de la metodología DEA en Colombia por actividad económica. Civilizar Ciencias Sociales y Humanas, 17(32), 133-160. Doi: $10.22518 / 16578953.823$
} 


\section{Introducción}

La competitividad ha sido y seguirá siendo un tema álgido para garantizar el bienestar social de una nación, ésta permite mejorar la calidad de vida de los ciudadanos a través de fuentes más dignas de empleo y una mayor cobertura en servicios básicos como la salud, la educación, la recreación y la vivienda. Es por ello, que se debe hacer uso de herramientas administrativas en las industrias para monitorear el desempeño organizacional en términos de eficiencia y potenciar así las estrategias competitivas.

Al respecto, Porter (2006) afirma que la gestión organizacional eficiente conlleva de manera agregada a generar mejores niveles de productividad en los sectores industriales del país. En sí, factores como la reestructuración, la reducción de costos y el aumento de la calidad son claves para generar ventaja competitiva, ya sea por una estrategia de liderazgo en costo al llegar con precios más bajos al mercado; otra estrategia de diferenciación de productos y servicios ante sus competidores; y estrategias de especialización ante un nicho de mercado identificado.

Por otra parte, el desarrollo de un país no solo se debe lograr a partir de la eficiencia en las operaciones productivas, sino también ésta debe implicar a la equidad. Después de todo, se ha establecido entre lo verdaderamente importante buscar la forma de usar los recursos de manera eficiente, con el propósito fundamental de conseguir los objetivos de la sociedad (Rodríguez, 2011).

Entonces, dentro de dichas herramientas administrativas el objeto de interés de la revisión bibliográfica del presente artículo, se encuentra en el Análisis Envolvente de Datos (DEA -Data Envelopment Analysis), el cual utiliza técnicas de programación lineal con el propósito de hacer la medición de las eficiencias técnicas relativas en las organizaciones, identificar las totalmente eficientes, y construir así lo que se conoce como la frontera eficiente. En sí, las organizaciones mal evaluadas en función de sus eficiencias se comparan con puntos totalmente eficientes de la frontera, lo que corresponde al nivel de eficiencia relativo (Fiallos, 2003).

Esta investigación es la continuación de la revisión bibliográfica sobre la aplicación de la metodología DEA en Colombia, puesto que ya se cuenta con una investigación realizada y publicada en revista científica por los mismos autores y que se titula "Revisión bibliográfica de la aplicación de la Metodología DEA en el ámbito educativo colombiano" (Cervera, Oviedo, \& Pineda, 2013); ésta investigación abarca la aplicación de la metodología DEA para medir eficiencias técnicas relativas en los procesos educativos, a nivel de grupos de investigación, en la formación de liderazgo en estudiantes, en el desempeño de instituciones educativas públicas, entre otros. Por lo tanto, la presente investigación se desarrolló a partir de la misma metodología en cuanto a la búsqueda, procesamiento y análisis de información recopilada; con el propósito de abarcar las demás actividades económicas sobre las cuales se ha aplicado la metodología DEA en Colombia.

Específicamente, lo que busca la revisión es determinar cómo ha sido el uso y la aplicación de la Metodología DEA a nivel nacional; para lo cual, se analizaron 58 investigaciones publicadas entre 1998 y 2014, agrupadas por las diferentes actividades económicas del CIIU'1.

Como resultado revelador de la revisión, se obtuvo que en los últimos 16 años el uso de la metodología DEA en Colombia se ha incrementado por parte de las organizaciones. Asimismo, se visualiza en la administración pública un mayor interés hacia este tipo de técnicas para evaluar su gestión.

La estructura de este artículo se compone de cuatro partes. La parte actual corresponde a la introducción, objetivo y alcance del estudio; la segunda parte, presenta la metodología utili- 
zada; posteriormente, se muestran los resultados de la revisión; y, por último, se exponen las conclusiones.

\section{Objetivo del estudio}

La finalidad del artículo es hacer una revisión bibliográfica de la producción intelectual asociada a la aplicación de la Metodología DEA en las diferentes actividades económicas en Colombia, aprender de la experiencia de otros investigadores e identificar oportunidades para la formulación de nuevos proyectos de investigación. Puntualmente, busca conocer los objetos de estudio asociados a dichas investigaciones, así como la forma en que se ha empleado la metodología.

\section{Metodología}

Las revisiones bibliográficas son utilizadas para analizar, sistematizar e integrar los resultados de diferentes investigaciones (Departamento Administrativo de Ciencia, Tecnología e Innovación, Colciencias, 2010). En general, permiten conocer lo ya investigado para no repetir el trabajo ya realizado por otros investigadores y tener un apropiado marco de referencia; documentar las contribuciones, los avances y las tendencias desarrolladas sobre un campo específico de conocimiento; conocer métodos de trabajo en casos similares; y obtener pruebas y conclusiones que contribuyan a las investigaciones en curso (Cegarra, 2004; Colciencias, 2010; Parra \& Toro, 2006).

El proceso inició con la ubicación de investigaciones a partir de los servicios de información de EBSCO, ProQuest, ScienceDirect y Scopus, entre otros; redes de revistas científicas, bibliotecas virtuales de algunas universidades, y repositorios digitales de diferentes entidades gubernamentales. Adicionalmente, se utilizó los motores de búsqueda de Google y Yahoo.

En los motores de búsqueda de dichas fuentes de datos, se utilizó el conector lógico
"Y" entre un grupo de palabras claves relacionado con DEA ("Análisis Envolvente de Datos", "DEA" y "eficiencia relativa") y otro con respecto al ámbito nacional ("Colombia", "colombiano").

Como criterios de inclusión, se consideraron los siguientes: investigación donde se aplique DEA, análisis aplicado a entidades nacionales, y documento publicado por entidades reconocidas (instituciones educativas, organizaciones gubernamentales o empresas).

Una vez filtrados los documentos, se vincularon a una carpeta compartida en Dropbox y se habilitó un documento en Excel Online para realizar su exploración, procesamiento y análisis a través de trabajo colaborativo. Como resultado, se revisaron 58 investigaciones.

\section{Exploración de las investigaciones.}

La exploración de las investigaciones se realizó a través de una matriz, donde se abstrajo las características generales de los documentos (título, tipo de documento, palabras clave, año y lugar de publicación, autores e institución que publica) y la información asociada al uso de la metodología DEA (sección CIIU, división CIIU, objeto de estudio, cobertura, tipo de DMU, modelo, objetivo de la medición de eficiencia, variables de entrada/salida, y herramientas de análisis). Posteriormente, se realizaron los cálculos estadísticos, se generaron las gráficas correspondientes y se analizaron los resultados.

\section{Características generales de los documentos.}

El tipo de documento más representativo corresponde a artículos (32 documentos), seguido de otros tipos de publicaciones realizadas por entidades públicas y privadas (11), trabajos de grado de pregrado (9) y posgrado (6) (gráfica 1). 
Gráfica 1.

Distribución por tipo de documento revisado

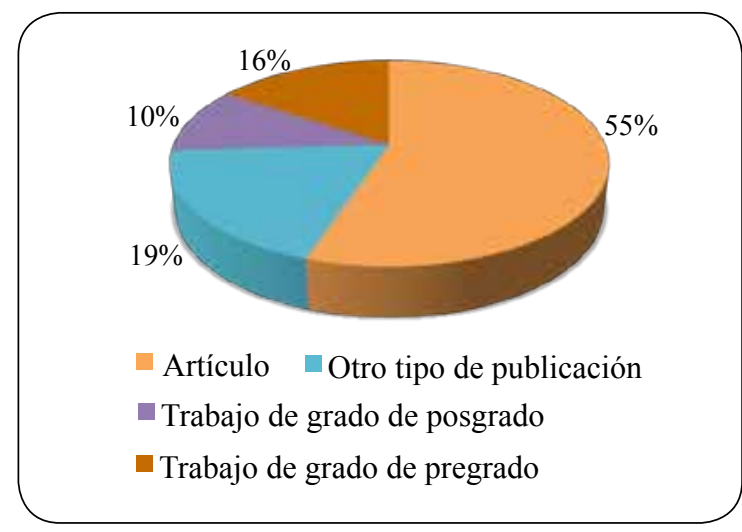

Fuente: elaboración propia.

Las universidades, demostrando la importancia de la aplicación de la metodología DEA para generar conocimiento, son las que más estudios han realizado (48 investigaciones). Así mismo, se evidencian 7 estudios por parte de entidades públicas, 2 de empresas privadas y 1 de entidad gremial (gráfica 2).
Gráfica 2.

Distribución por tipo de institución que publica

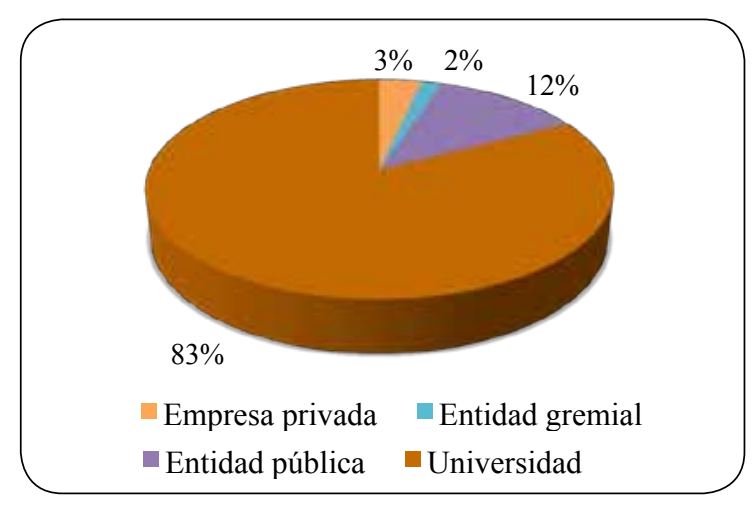

Fuente: elaboración propia.

Respecto a la frecuencia de las publicaciones, se encontraron estudios desde el año 1998, incrementándose en el 2003 (4 investigaciones), y con un máximo en el 2011 (8). En general, existe una tendencia positiva (gráfica 3) hacia el uso y aplicación para resolver problemas y proponer optimizaciones en los sectores de la economía colombiana.

Gráfica 3.

Tendencia de publicaciones

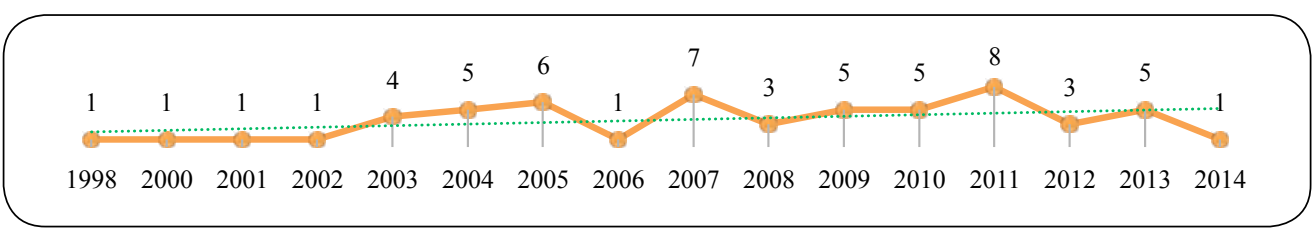

Fuente: elaboración propia.

Asimismo, se tiene que las ciudades que más investigan sobre los sectores económicos empleando DEA son: Bogotá (41 investigaciones), Pereira (4 investigaciones), Cali (3 investigaciones), y Cartagena (3 investigaciones) (gráfica 4).

Gráfica 4.

Frecuencia de publicaciones por lugar de origen

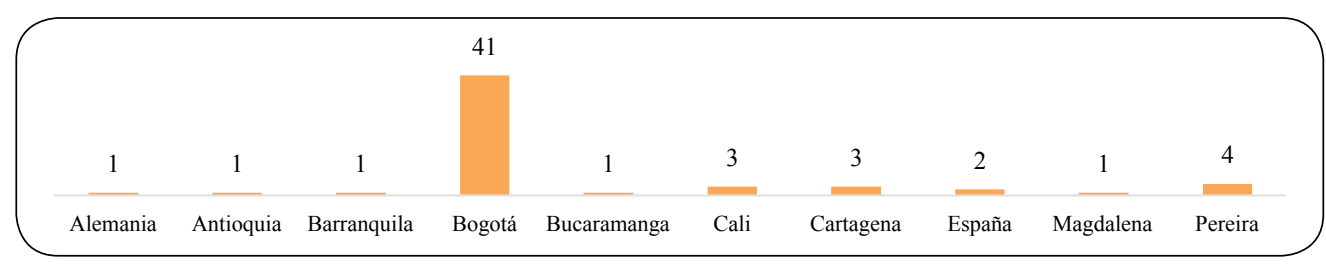

Fuente: elaboración propia. 
Finalmente, las palabras claves más utilizadas por los autores de los documentos fueron: "eficiencia", "Análisis Envolvente de
Datos", "Modelo DEA, entradas y salidas", "productividad y competitividad" y "recursos y optimizaciones" (gráfica 5).

\section{Gráfica 5.}

\section{Frecuencia de categorías de palabras claves}

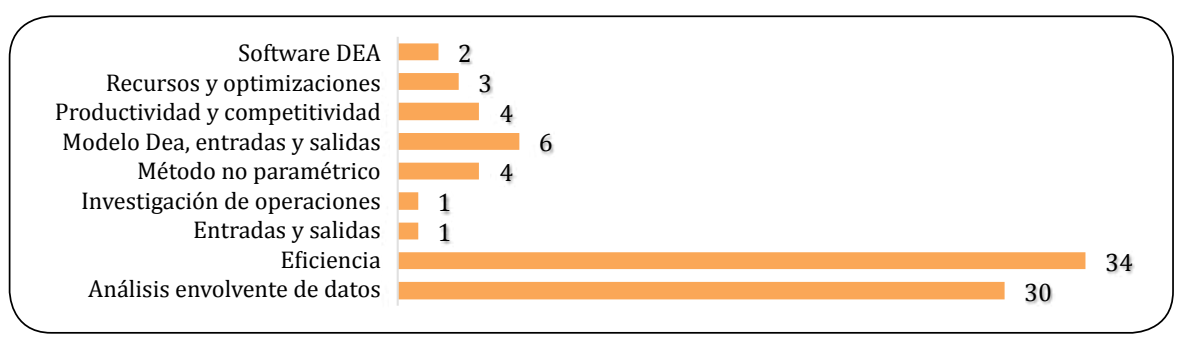

Fuente: elaboración propia.

\section{Hallazgos en la aplicación de DEA en las actividades económicas en Colombia}

En esta sección se presenta cómo se ha abordado la metodología DEA en las diferentes secciones del CIIU.

\section{Secciones del CIIU abordadas por la metodología DEA.}

Al abordar la aplicación y utilización de la metodología DEA en las actividades econó- micas, se tiene que la sección más representativa es la $\mathrm{O}$, correspondiente a "administración pública y defensa, planes de seguridad social de afiliación obligatoria" (13 investigaciones), seguido de la sección A "agricultura, ganadería, caza, silvicultura y pesca" (7 investigaciones), la sección K "actividades financieras y de seguros" (7 investigaciones), la sección Q "actividades de atención de salud humana y de asistencia social" (6 investigaciones), y la sección D "suministro de electricidad, gas, vapor y aire acondicionado" (gráfica 6). Lo anterior, confirma que el sector público es el que más emplea la metodología.

Gráfica 6.

Frecuencia de investigaciones por secciones CIIU

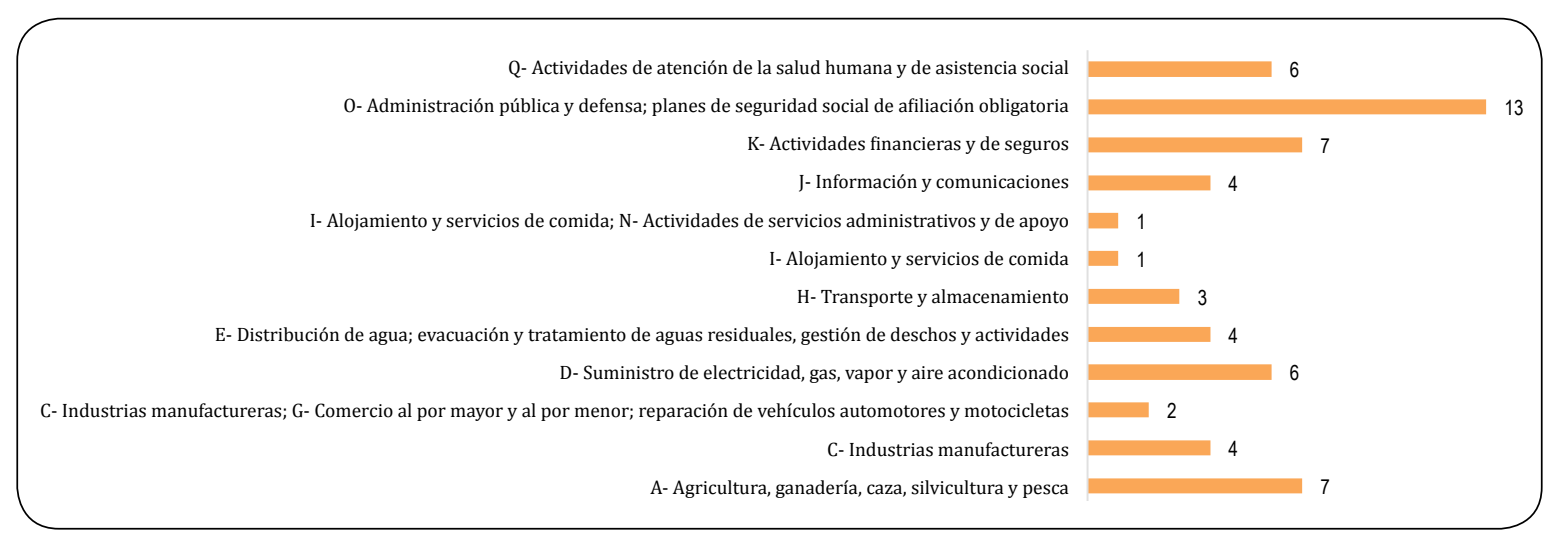

Fuente: elaboración propia.

Al relacionar la frecuencia de documentos por sección del CIIU y el tipo de institución que las realiza, las universidades son las que más investigan, seguido de las entidades públicas (gráfica 7). 
Gráfica 7.

Frecuencia de investigaciones por secciones CIIU y tipo de institución que publica

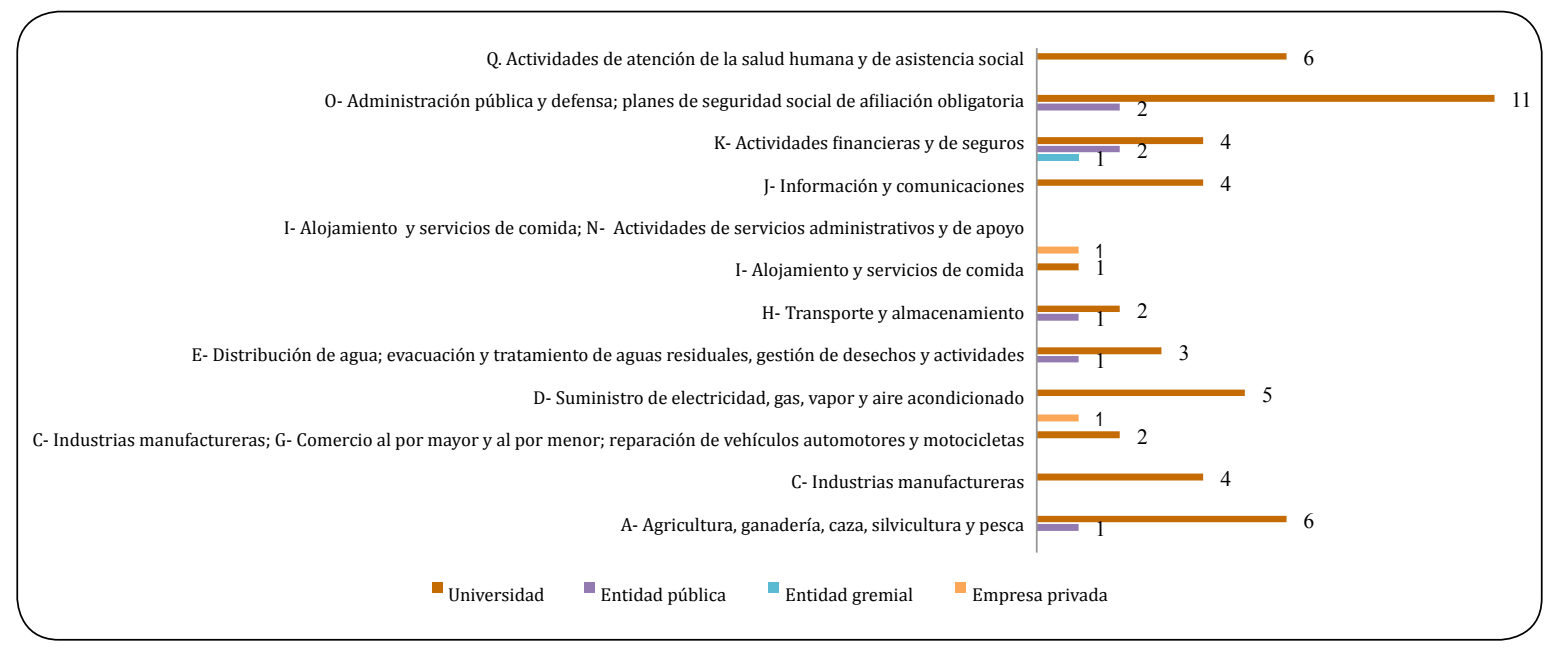

Fuente: elaboración propia.

Complementando, en la gráfica 8 se presenta el desagregado de cada sección.

Gráfica 8.

Frecuencia de investigaciones por divisiones de las secciones CIIU

Sección A - Agricultura, ganadería, caza, sivicultura y pesca

01 - Agricultura, ganadería, caza y actividades de servicios conexas

Sección C - Industrias manufactureras

15 - Curtido y recurtido de cueros; fabricación de calzado; fabricación de artículos de viajes; maletas, bolsos de mano y artículos similares, y fabricación de artículos de talabartería y guanicionería; adobo y teñido de pieles

20 - Fabricación de sustencias y productor químicos 13 - Fabricación de productos textiles

10 - Elaboración de productos alimenticios

24 - Fabricación de productos metalúrgicos básicos

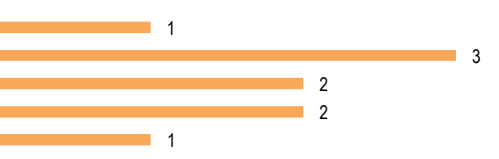

Sección D - Suministro de electricidad, gas, vapor y aire

35 - Suministro de electricidad, gas, vapor y aire

Sección E - Distribución de agua; evacuación y tratamiento de aguas residuales...

36 - Captación, tratamiento y distribución de agua

Sección G - Comercio al por mayor y al por menor...

45 - Comercio, mantenimiento y reparación de vehículos

Sección H - Transporte y almacenamiento

52 - Almacenamiento y actividades complementarias al transporte 49 - Transporte terrestre; transporte por tuberías 50 - Transporte acuático

Sección I - Alojamiento y servicios de comida

55 - Alojamiento

Sección J - Información y comunicaciones

62 - Desarrollo de sistemas informáticos (planificación, análisis, diseño, programación, pruebas), consultoría informática y actividades relacionadas 58 - Actividades de edición 61 - Telecomunicaciones 
Sección K - Actividades financieras y de seguros

65 - Seguros (incluso el reaseguro), seguros sociales y fondos de pensiones, excepto la seguridad social 64 - Actividades de servicios financieros, excepto las de seguros y pensiones

Sección N - Actividades de servicios administrativos y de apoyo

79 - Actividades de las agencias de viajes, operadores

Sección 0 - Administración pública y defensa; planes de seguridad social...

84 - Administración pública y defensa; planes de seguridad social de afiliación obligatoria

Sección Q - Actividades de atención de la salud y de asistencia social

Fuente: elaboración propia.

\section{Caracterización de las DMU por sección CIIU.}

Cada unidad tomadora de decisión en DEA se denomina DMU (Decision Making Units), la cual considera múltiples entradas (inputs, recursos o factores de producción) y múltiples salidas (outputs o productos) (Sepúlveda, 2014). Con estas entradas y salidas se busca medir la eficiencia técnica relativa, que refleja la habilidad de una DMU para obtener el máximo nivel de producción con unos recursos dados, esta eficiencia es calculada para varias DMU que emplean los mismos insumos para producir los mismos productos (Fiallos, 2003).

\section{Tipos de DMU analizadas.}

Las DMU más utilizadas en las investigaciones fueron: empresas (16 en ocho secciones), municipios (7 en dos secciones), hospitales (4 en una sección), departamentos del territorio nacional (4 en dos secciones) y bancos (3 en una sección) (gráfica 9).

\section{Gráfica 9.}

\section{Distribución del tipo de DMU por sección CIIU}

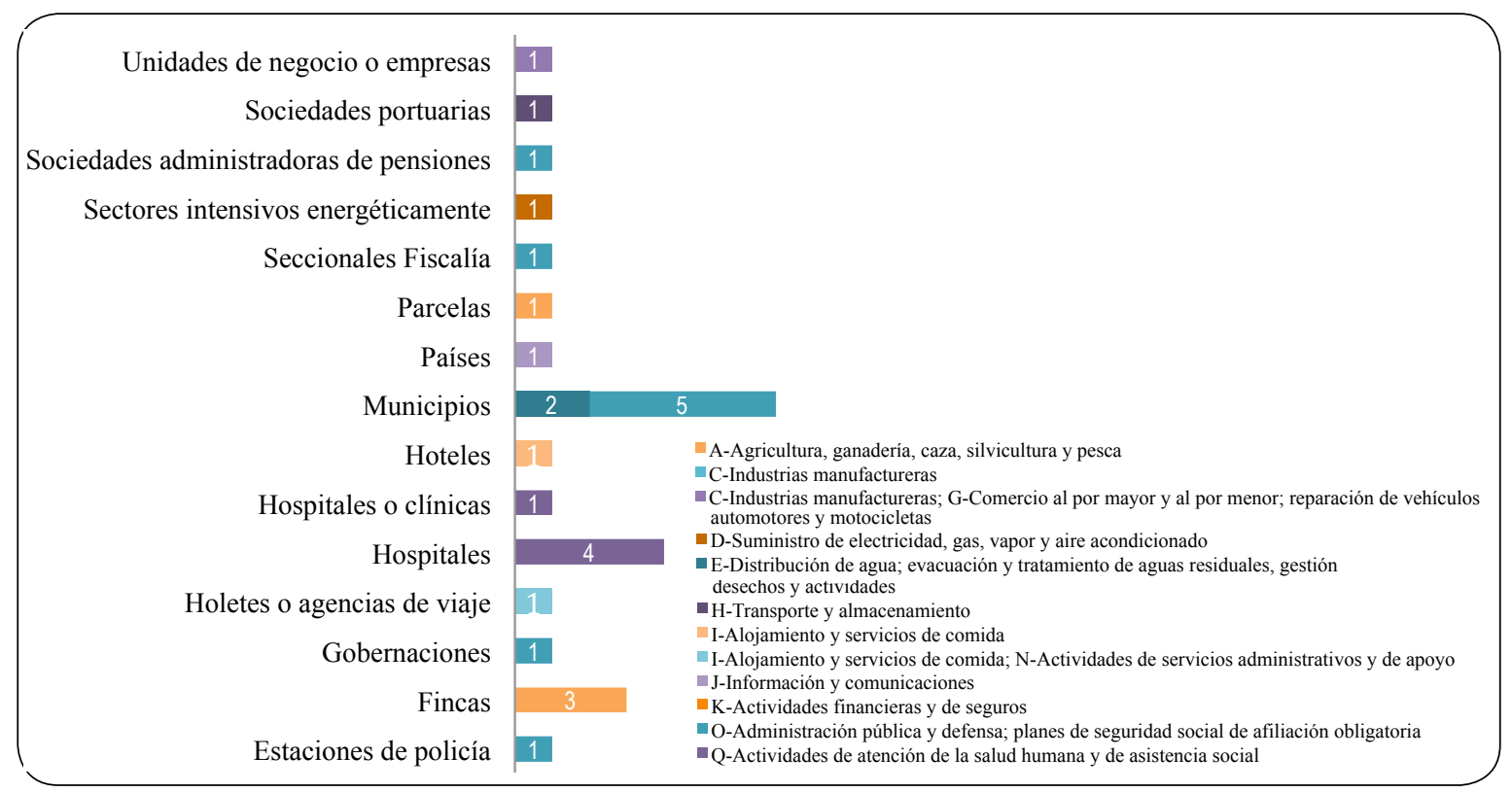




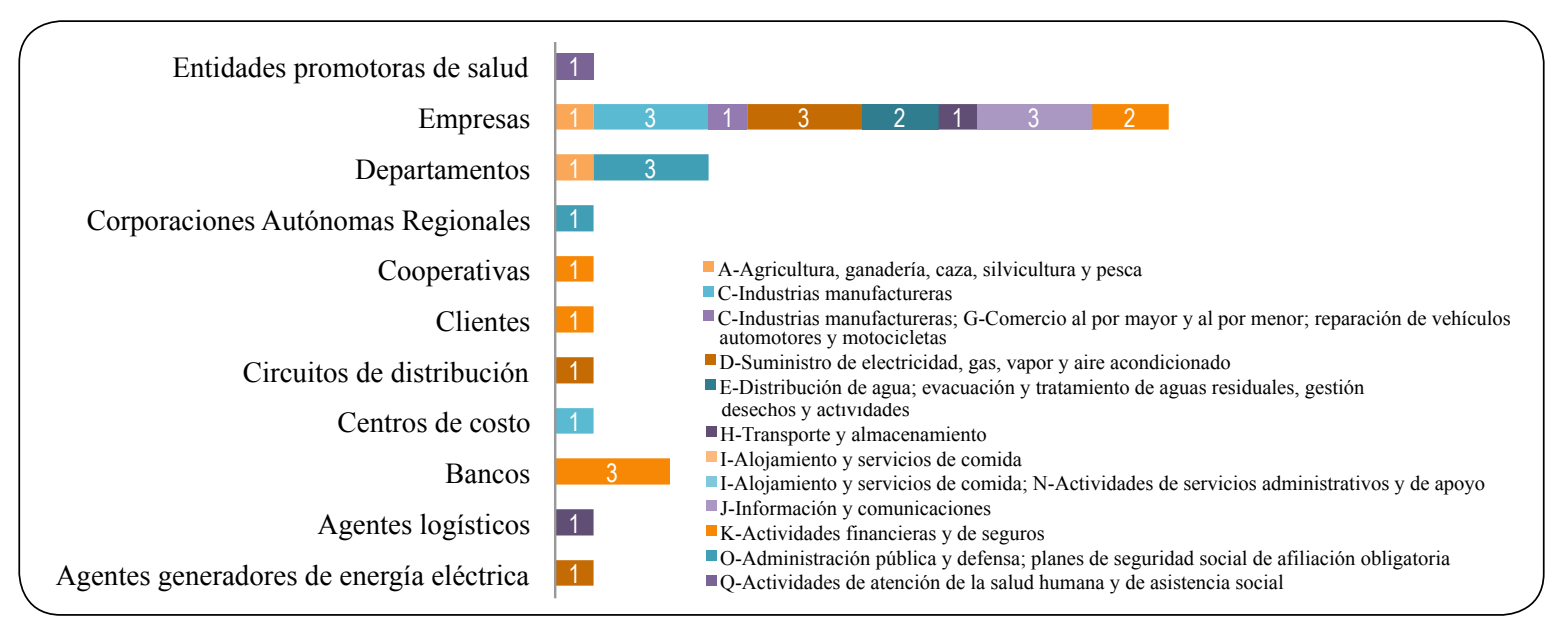

Fuente: elaboración propia.

\section{Cobertura del estudio.}

La cobertura del estudio corresponde al alcance de las DMU, es decir, si la comparación de eficiencia se hizo dentro de una organización o a nivel de localidad, municipio, país u otro. En las investigaciones, (Gráfica 10), el nivel país fue el de mayor empleo (74\%).

Gráfica 10.

Distribución de la cobertura por estudio

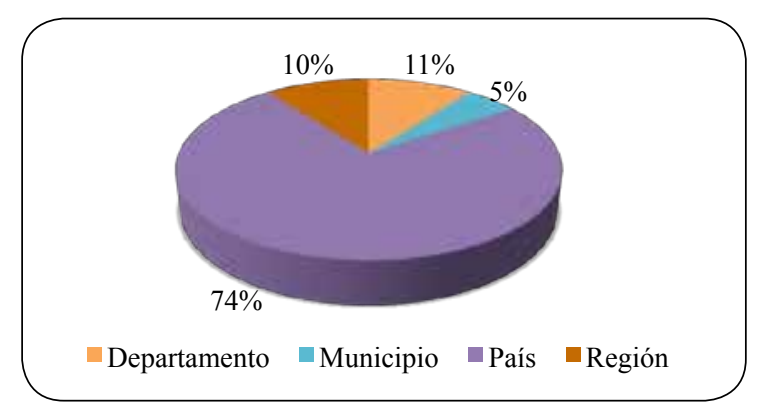

Fuente: elaboración propia.

\section{Análisis envolvente de datos en las secciones del CIIU.}

Teniendo en cuenta el objetivo de la aplicación de DEA en las actividades económicas, a continuación se presenta tanto los modelos como las variables que se emplearon.

\section{Eficiencia evaluada.}

Se identificaron ocho objetivos de medición de eficiencia en los documentos anali- zados, estos fueron: manejo de recursos naturales, gestión agropecuaria, gestión pública, gestión organizacional, gestión industrial, producción, planeación estratégica y manejo de recursos financieros (gráfica 11). El objetivo más relevante fue el de gestión organizacional (17 estudios), seguido por gestión pública (10 estudios) y gestión industrial (9 estudios). 
Gráfica 11.

Frecuencia de los objetivos de medición de eficiencia

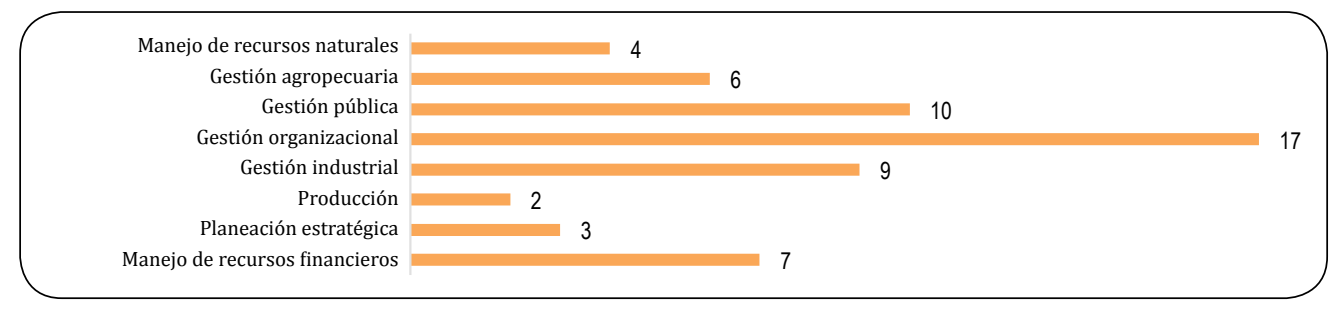

Fuente: elaboración propia.

Por otra parte, con respecto a la aparición de la frecuencia de los objetivos de medición de eficiencia por sección CIIU según gráfica 12; el más representativo es la gestión organizacional que aparece en nueve secciones del CIIU con 17 investigaciones en total: actividades financieras y de seguros (4 aplicaciones); administración pública y defensa, planes de seguridad social de afiliación obligatoria (3); actividades de atención de la salud humana y de asistencia social (2); transporte y almacenamiento (2); industrias manufactureras (2); información, comunicaciones, alojamiento y servicios de comida (1). Asimismo, la gestión pública en tres secciones con 10 investigaciones en total: administración pública y defensa, planes de seguridad social de afiliación obligatoria (6); actividades de atención de la salud humana y de asistencia social (3), y agricultura, ganadería, caza, silvicultura y pesca (1).

\section{Gráfica 12.}

Frecuencia de los objetivos de medición de eficiencia por sección CIIU

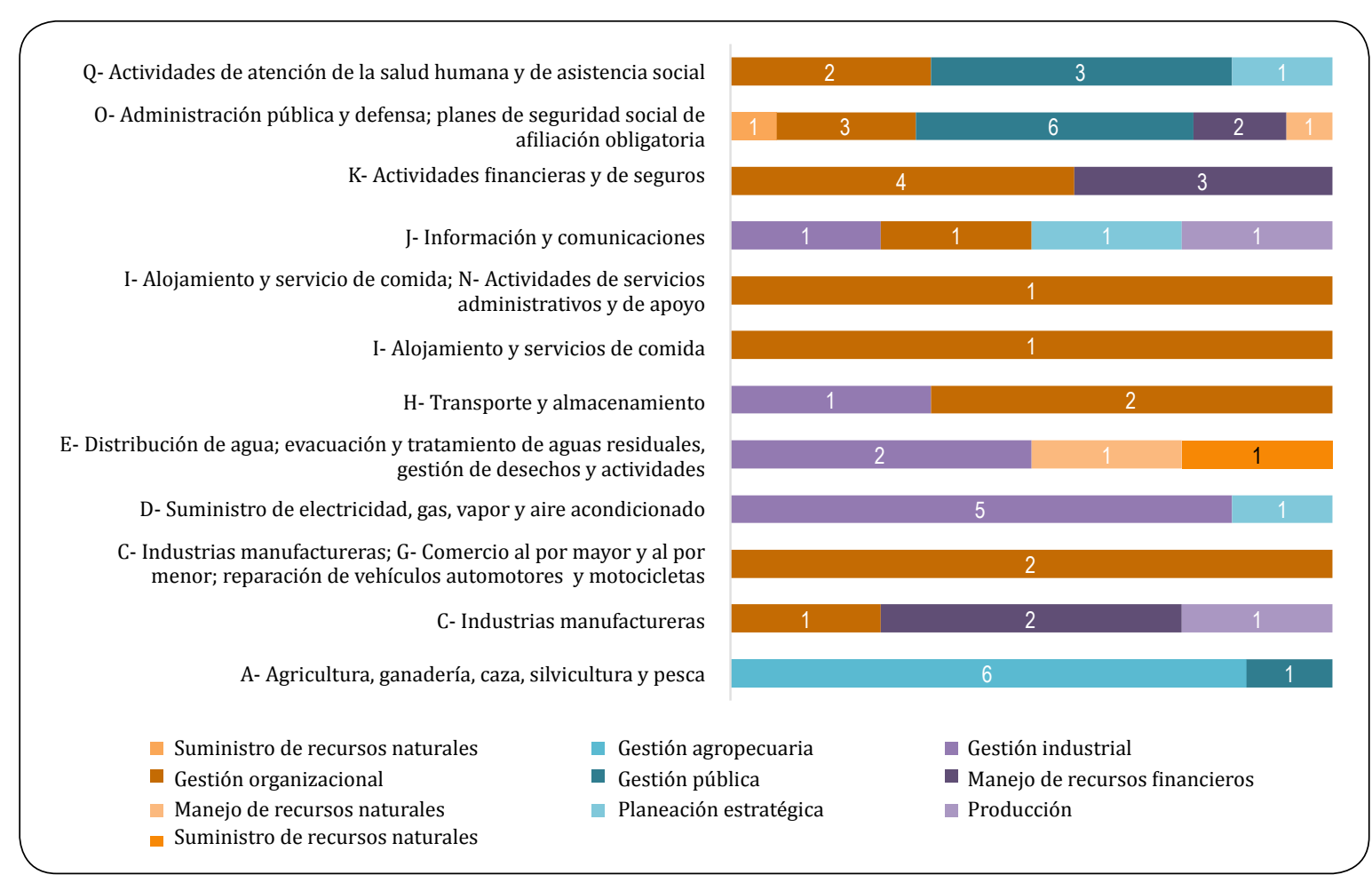

Fuente: elaboración propia. 


\section{Modelos DEA.}

En los modelos usados en DEA, se tiene el CCR (Charnes, Cooper \& Rhodes) y el BCC (Banker, Charnes \& Cooper), que se construyen por proyección radial (el primero con rendimientos constantes a escala [CRS] $\mathrm{y}$ el segundo con rendimientos variables a escala [VRS]). Ambos pueden ser orientados a entradas o salidas; en los orientados a las entradas, estas pueden ser proporcionalmente reducidas y aún producir el mismo nivel de salidas; y en los orientados a las salidas, estas pueden ser proporcionalmente aumentadas dados los niveles de las entradas existentes (Alfonso \& Gutiérrez, 2007).

En particular, en las 58 investigaciones; 57 emplearon su respectivo modelo de DEA elegidos entre los cuatro modelos identificados en toda la revisión bibliográfica (CCR; BCC; $\mathrm{SBM} \&$ \&CR-BCC); estos modelos fueron enfocados tanto a las entradas como a las salidas (gráfica 13).

Gráfica 13.

Frecuencia de uso de modelos DEA

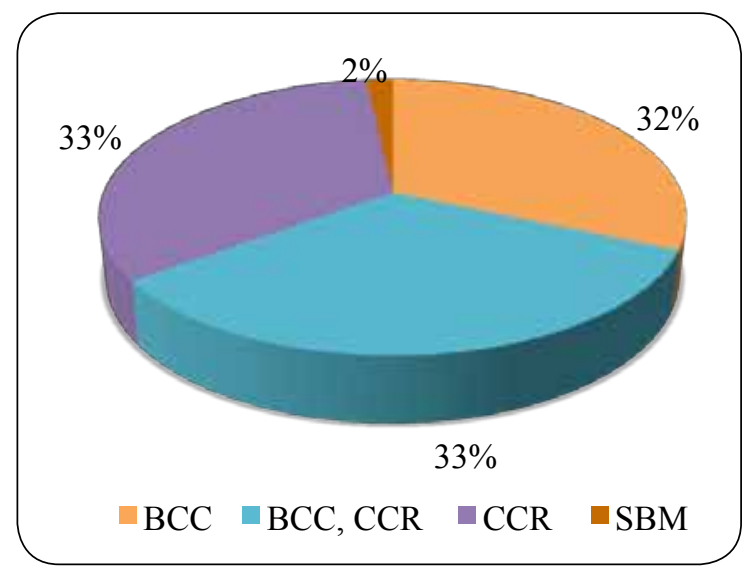

Fuente: elaboración propia.

De esta manera, se establece que la orientación de los estudios, en los que lo explicitaron, se encauza más hacia las entradas (37 aplicaciones) que hacia las salidas (19 aplicaciones).

\section{Variables contempladas en los mo- delos.}

DEA es un método de estimación para construir la frontera de eficiencia, lo que requiere relacionar las entradas con las salidas a través de los objetivos (López \& Fernández, 2009). Es por ello, y teniendo en cuenta los ocho objetivos identificados, que a continuación se presenta el análisis de variables para establecer nexos o discrepancias por sección.

\section{Sección A: Agricultura, ganadería, caza, silvicultura y pesca.}

Para la primera sección CIIU los objetivos identificados fueron gestión agropecuaria (16) y gestión pública (8), lo que ratifica el que la Metodología DEA pretende lograr una eficiente gestión (Maza, Navarro, \& Puello, 2012).

Puntualmente, Luna (2012) afirma que el stock de capital, el trabajo, el consumo y la energía eléctrica son los factores determinantes de la eficiencia técnica del sector. Complementando, y frente al crecimiento de las organizaciones, Sepúlveda (2014) considera que la estructura de costos, los gastos operacionales, los ingresos operacionales y el consumo, son los factores que más incidencia tienen. A su vez, Montoya y Soto (2010), puntualizan que el control de los productores sobre sus recursos o insumos y su capacidad de planta, es determinante para obtener mayores niveles de producción. Lo anterior, confirma que DEA en el sector agrícola se utiliza para optimizar los insumos, incrementar la capacidad de producción y por ende buscar mayor competitividad.

En cuanto a los estudios centrados en el sector pecuario, la búsqueda se enfoca en la optimización de la nutrición de los animales para la mejora de la producción de carne, crías y leche (Gamarra, 2004). De igual manera, Oviedo y Rodríguez (2011) hacen énfasis en la utilización óptima de insumos como la nutrición, el mantenimiento y el ordeño, para 
mejorar el nivel de eficiencia en la producción de leche, carne y crías.

Frente a las variables de entrada (gráfica 14), se tienen los gastos operacionales, los activos y los recursos; donde las unidades empresariales, como los grandes productores, campesinos o minifundistas, deben emplear la cantidad óptima de insumos o recursos para obtener una mayor producción y menores costos de producción. No obstante, los pequeños productores no lo logran (Perdomo \& Mendieta, 2007).

\section{Gráfica 14.}

Variables de entrada para sección A según los objetivos de gestión agropecuaria y gestión pública

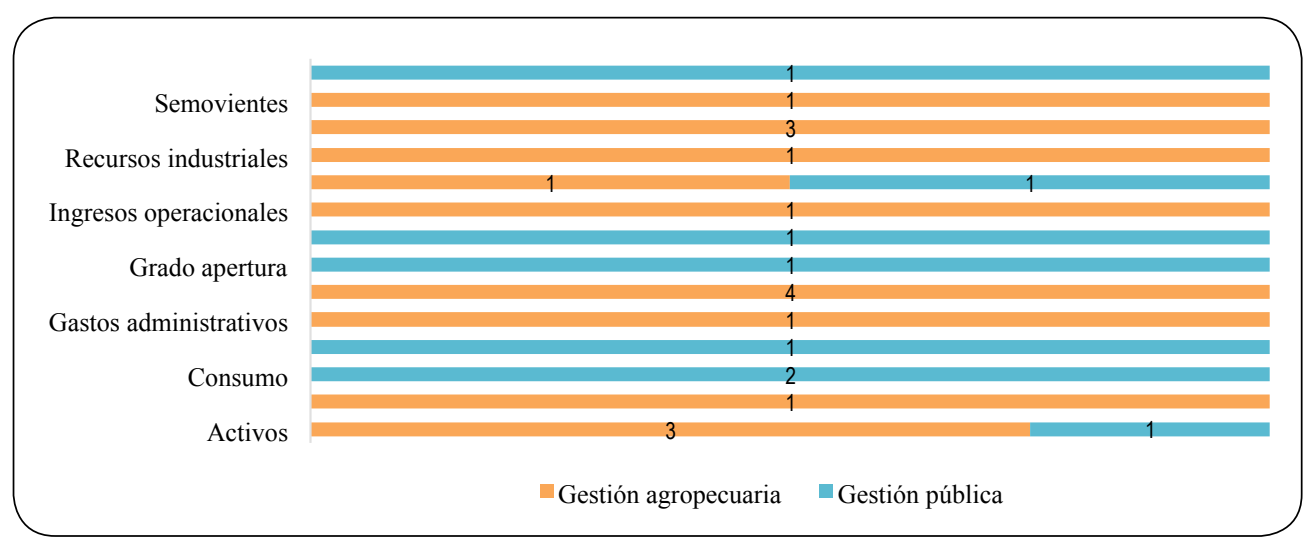

Fuente: elaboración propia.

En las salidas (gráfica 15), el objetivo de gestión agropecuaria es el más representativo en las investigaciones realizadas (18 investigaciones) en las actividades económicas de la sección A: Agricultura, ganadería, silvicultura, caza y pesca.

Al respecto, Perdomo y Mendieta (2007) afirman que la eficiencia técnica y asignativa son análisis relativos, empleados en procesos productivos para conocer el máximo nivel producido y cantidad óptima utilizada de insumos acordes con sus precios, por lo que se puede inferir que cuanto más productivas sean las empresas agropecuarias mayor será el nivel de rentabilidad y tendrán mejores oportunidades de mercado.

Gráfica 15.

Variables de salida para sección A según los objetivos de gestión agropecuaria y gestión pública

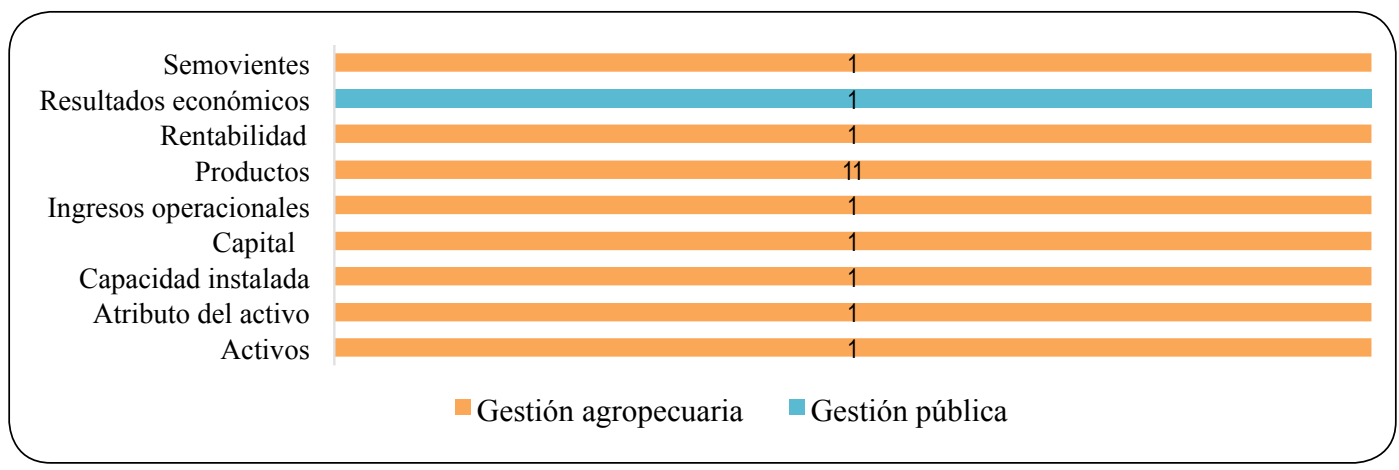

Fuente: elaboración propia. 
Sección C-G: Industrias manufactureras y comercio al por mayor y al por menor; reparación de vehículos automotores y motocicletas.

Las secciones $\mathrm{C}-\mathrm{G}$ se abordaron en seis estudios, donde los objetivos de gestión organizacional y manejo de recursos financieros son los más representativos.

Específicamente, Rodríguez (1998) afirma que las empresas que no tienen buenos márgenes operacionales y/o netos no son organizaciones eficientes bajo el esquema de cálculo de razones financieras, aunque se encuentren sobre una frontera eficiente. Asimismo, Aristizabal y Salazar (2011), indican que la ineficiencia de las empresas se debe a un exceso de gastos de administración y un bajo nivel de razón corrien- te, donde la liquidez es menor para mejorar su proceso productivo.

Lo anterior, presenta la importancia de los ingresos operacionales y sus activos representados en propiedades, planta, equipos y patrimonio; e implica que a medida que las empresas aumentan los gastos administrativos y los gastos de ventas, existe una menor utilidad bruta y una razón corriente, y por consiguiente las empresas son menos eficientes en sus indicadores financieros.

Luego, en las secciones $\mathrm{C}$ y $\mathrm{C}-\mathrm{G}$ se tiene como objetivo apreciable la gestión organizacional, que está presente en diez investigaciones que se pueden observar en las gráficas 16 y 17, tal como lo afirma Cayon (2001), donde expone que para medir la rentabilidad real de una compañía como una función del costo de capital, un factor relevante son los activos.

Gráfica 16.

Variables de entrada para la sección $C$ según los objetivos de gestión organizacional, manejo de recursos financieros y producción

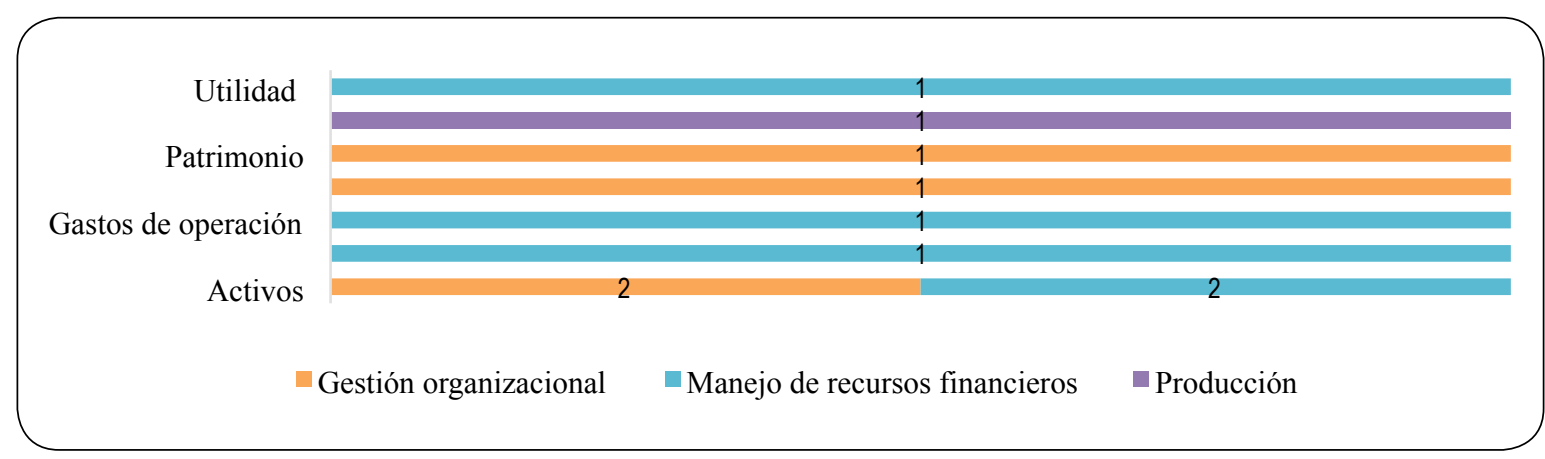

Fuente: elaboración propia.

Gráfica 17.

Variables de entrada para la sección C-G según el objetivo de gestión organizacional

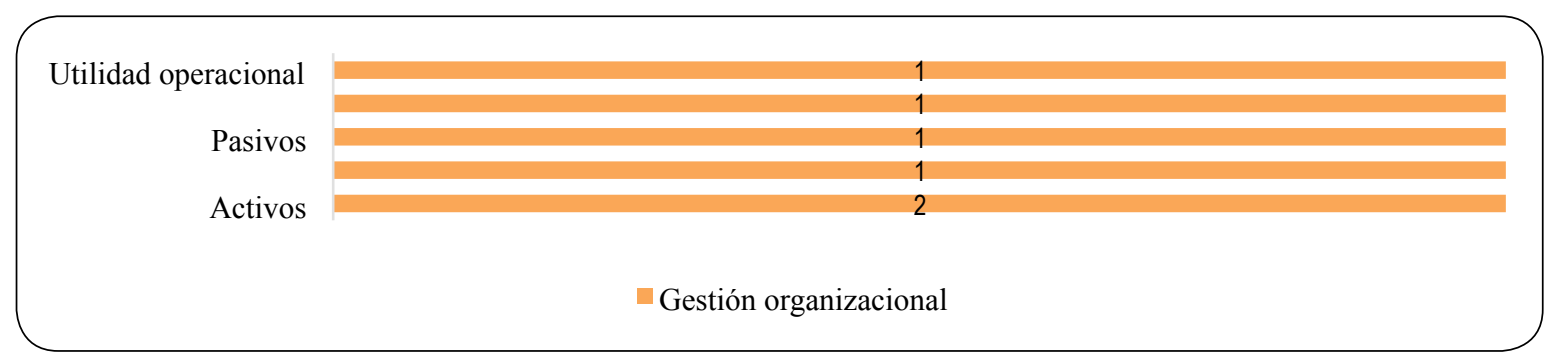

Fuente: elaboración propia. 
En cuanto a las salidas (gráfica 18 y 19), se tiene que la utilidad neta, ingresos operacionales, patrimonio y activo corriente están relacionados con el objetivo de gestión organizacional. Esto se debe, a que las organizaciones relacionadas con estas dos secciones tienen como fin manejar sus recursos eficientemente, basados en una comparación frente a la información que aporta un análisis de indicadores financieros, tales como los márgenes operacionales y neto (Rodríguez, 2003a; 2003b).

\section{Gráfica 18.}

Variables de salida para sección C según los objetivos de gestión organizacional, manejo de recursos financieros y producción

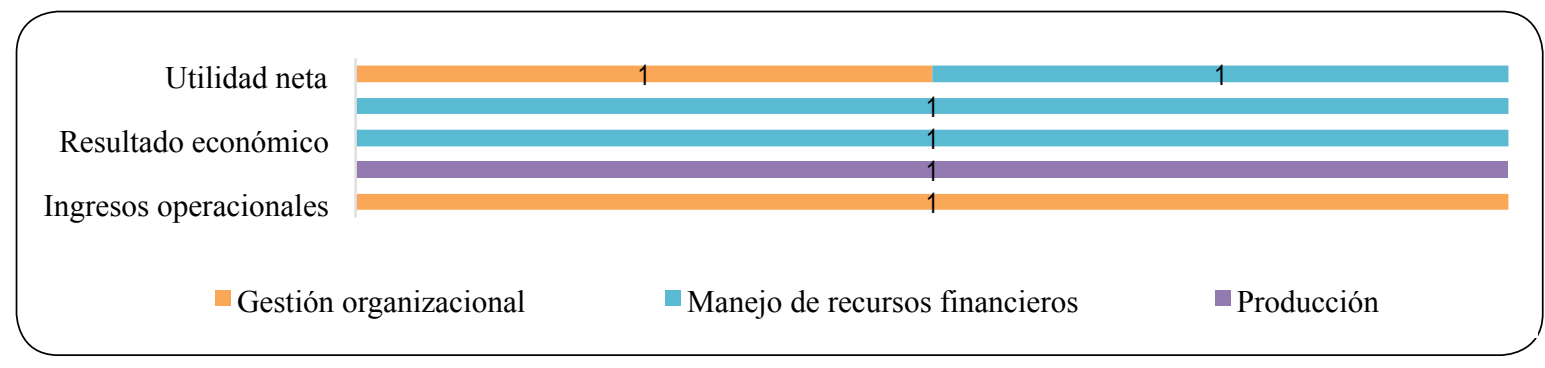

Fuente: elaboración propia.

Gráfica 19.

Variables de salida para sección C-G según el objetivo de gestión organizacional

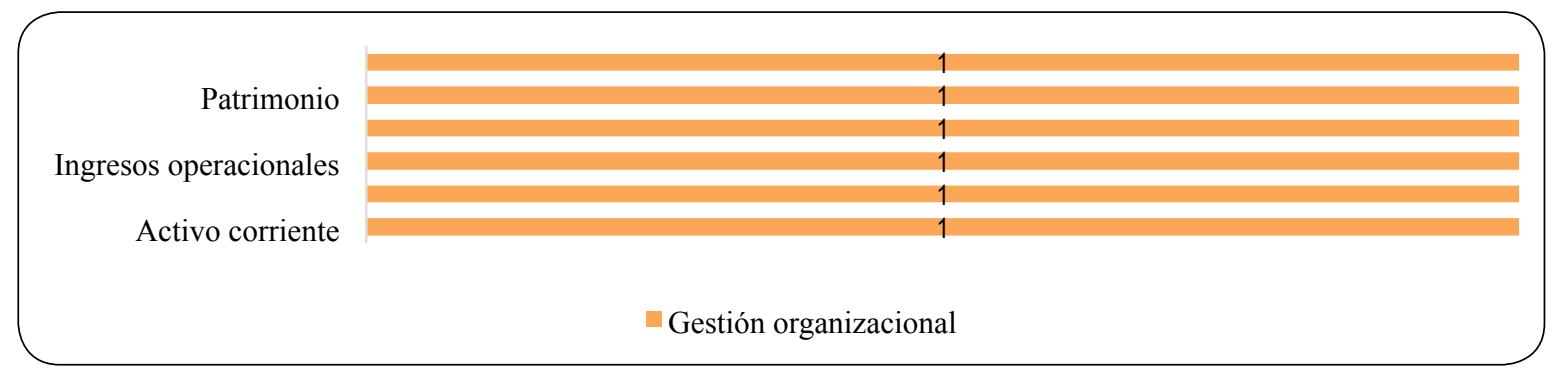

Fuente: elaboración propia.

Sección D: Suministro de electricidad, gas, vapor y aire acondicionado.

El objetivo más relacionado con esta sección es la planeación estratégica, representado en seis investigaciones. Esto indica, que es un proceso fundamental dentro de las organizaciones, para analizar y evaluar su situación presente, su competitividad y decidir sobre el direccionamiento de la misma. En general, las empresas del sector energético y eléctrico han experimentado cambios desde el año 1990, por las dificultades económicas que han enfrentado, haciendo que establezcan estrategias de competencia que han incidido fundamentalmente en su funcionamiento y en la eficiencia de sus operaciones (García, 2005; Guio \& Monroy, 2003).

Ahora bien, el Gobierno, con la expedición de las leyes 142 y 143 de julio de 1994 estableció un nuevo régimen para los Servicios Públicos Domiciliarios y el Sector Eléctrico; en el cual las actividades del sector se deben regir por principios de eficiencia, calidad, continuidad, adaptabilidad, neutralidad, solidaridad y equidad, donde se promueva la libre competencia. Y es precisamente sobre los principios de eficiencia y calidad que algunos autores han expuesto: 
[...] la eficiencia en la prestación del servicio de electricidad debe verse reflejada en costos de prestación, que representen valor económico del mismo, impidiendo transmitir a los usuarios las ineficiencias de la operación. Por lo tanto, la energía, como un factor productivo, es importante dentro de las estructuras de producción y es un elemento clave dentro del desarrollo tecnológico (Pardo \& Cotte, 2011, pp. 49).

Con DEA, se busca establecer cómo utilizar los recursos suficientes, para adelantar los programas de inversión necesarios para garantizar la mejora en la calidad del servicio (González, 2010). De hecho, se traduce en que las empresas pertenecientes a este sección están forzadas positivamente a evolucionar para mejorar, perdurar y prosperar en un ambiente de negocios frente a la competencia global; gestionando estratégicamente al revelar las fortalezas y debilidades, con el fin de articular la información necesaria, precisa y a tiempo, para cumplir las necesidades y los requerimientos de todos los clientes (García, 2005).

En conclusión, las variables de entrada asociadas a recursos humanos, recursos tecnológicos, recursos industriales y recursos materiales son las más representativas en esta sección (gráfica 20).

Gráfica 20.

Variables de entrada para sección D según los objetivos de gestión industrial y planeación estratégica

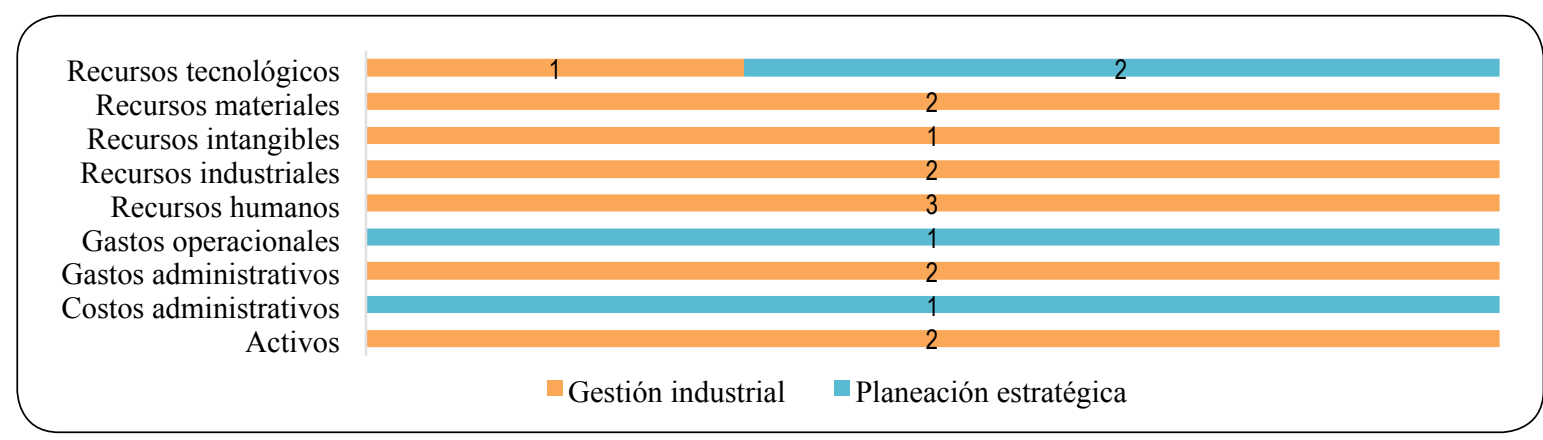

Fuente: elaboración propia.

Frente a las salidas (gráfica 21), las organizaciones relacionadas con esta sección muestran dos estudios enfocados en la prestación de servicios públicos, donde se han visto evocadas a optimizar sus procesos, para obtener la rentabilidad esperada de los inversionistas y lograr un control de pérdidas y la calidad del servicio (Rojas, 2011; Tolosa, 2013).

\section{Gráfica 21.}

Variables de salida para sección D según los objetivos de gestión industrial y planeación estratégica

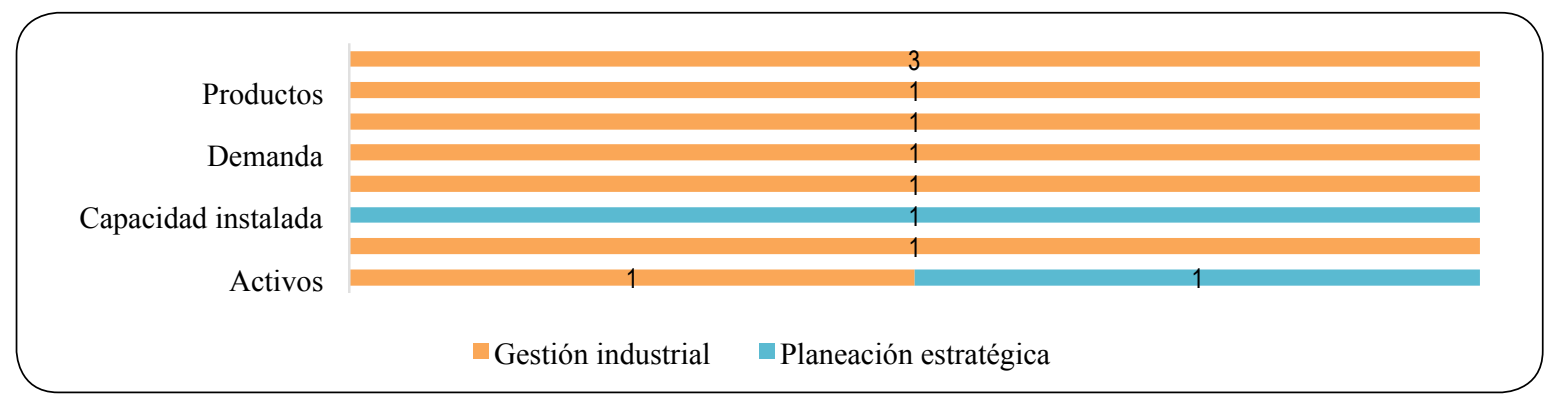

Fuente: elaboración propia. 


\section{Sección E: Distribución de agua; evacuación y tratamiento de aguas residuales, gestión de desechos y ac- tividades de saneamiento ambiental.}

Esta sección muestra cuatro estudios relacionados con el objetivo de gestión industrial. Aquí, la industria de agua potable en Colombia al igual que en otros sectores, tiene como objetivo garantizar la prestación de un servicio de mejor calidad, teniendo una gestión eficiente de sus recursos (Urrego, 2010).

Con relación al servicio de agua potable:

[...] en la Constitución Política [...] se estableció que los servicios públicos son inheren- tes a la finalidad social del Estado y es deber de sus instituciones asegurar su prestación eficiente a todos los habitantes del territorio nacional; establece, además, que un objetivo fundamental es la solución de necesidades insatisfechas en saneamiento ambiental y agua potable (Maza et al., 2012, pp. 59).

Las variables de entrada (gráfica 22), incluyen los costos de operación, como la más representativa, lo que permite concluir que las empresas prestadoras de este servicio tienen dentro de sus objetivos el cubrimiento de los costos eficientes de prestación del servicio, buscando minimizar el insumo de gastos administrativos para generar un producto y un nivel de gestión eficiente (Comisión de Regulación de Agua Potable y Saneamiento Básico, CRA, 2005).

Gráfica 22.

Variables de entrada para sección $E$ según los objetivos de gestión industrial y manejo de recursos naturales

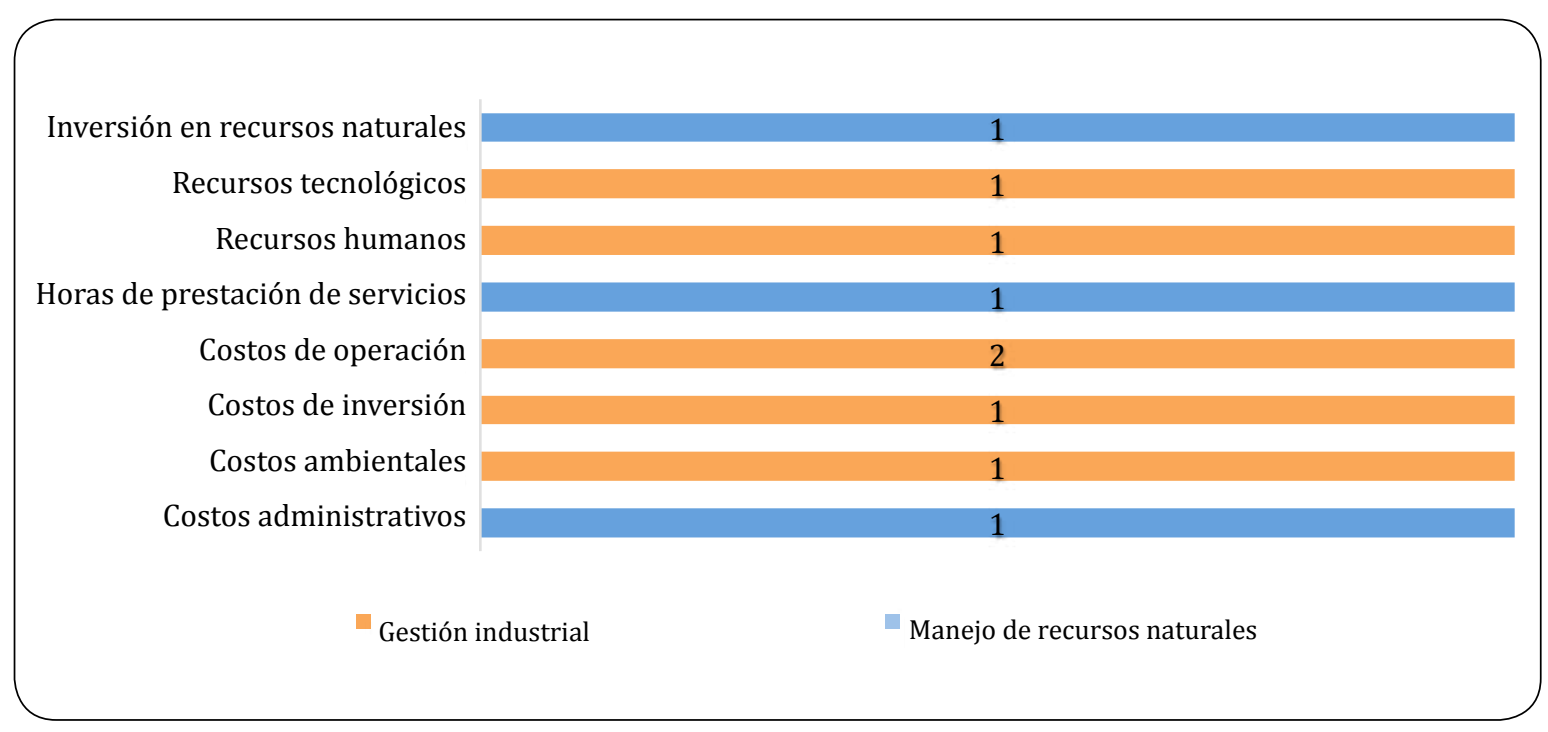

Fuente: elaboración propia.

En cuanto a las salidas (gráfica 23), la más relevante es la de clientes que hacen uso del servicio de agua potable y quienes manifiestan satisfacción en la entrega del servicio cuando las compañías proveedoras de agua manejan precios razonables en sus facturas y un servicio sin interrupciones. No obstante, no es posible garantizar a los usuarios del servicio (clientes) estas dos variables por factores ambientales que no son controlables; a pesar de esto se busca dar un paso importante hacia la calidad y excelencia del sector del agua colombiano (Cunha \& Garzón, 2007). 
Gráfica 23.

Variables de salida para sección $\mathbf{E}$ según los objetivos de gestión industrial y manejo de recursos naturales

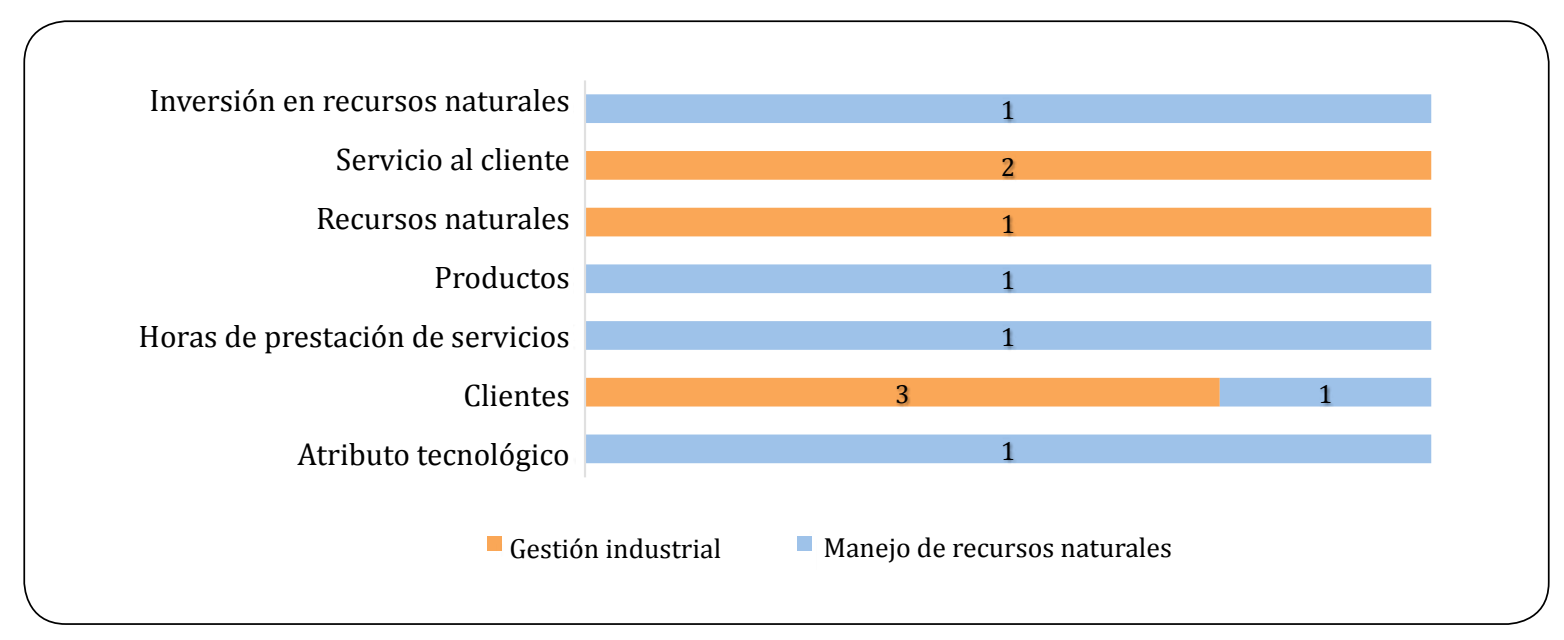

Fuente: elaboración propia.

\section{Sección H: Transporte y almacena- miento.}

La gestión organizacional se consideró como el principal objetivo relacionado con las investigaciones de esta sección, con tres estudios, donde las empresas portuarias asociadas a esta sección analizan la eficiencia de la tecnología disponible y uso del tiempo a los costos declarados, como factores claves para medir su gestión y orientar el mercado de la infraestructura portuaria hacia los intereses de la sociedad y del sistema económico en su globalidad (Duncan, 2008).
Asimismo, las empresas transportadoras de gas, miden su gestión respecto a la evaluación de la eficiencia de los gastos en administración, operación y mantenimiento (AO\&M) (Comisión de Regulación de Energía y Gas, CREG, 2000).

En las variables de entrada (gráfica 24), estas incluyen los recursos tecnológicos, los ingresos operacionales y los gastos operacionales, lo que permite concluir que las empresas asociadas a esta sección se clasifican en razón de su índice de eficiencia tanto en infraestructura tecnológica como en el manejo eficiente de sus gastos operacionales.

Gráfica 24.

Variables de entrada para sección $H$ según los objetivos de gestión industrial y gestión organizacional

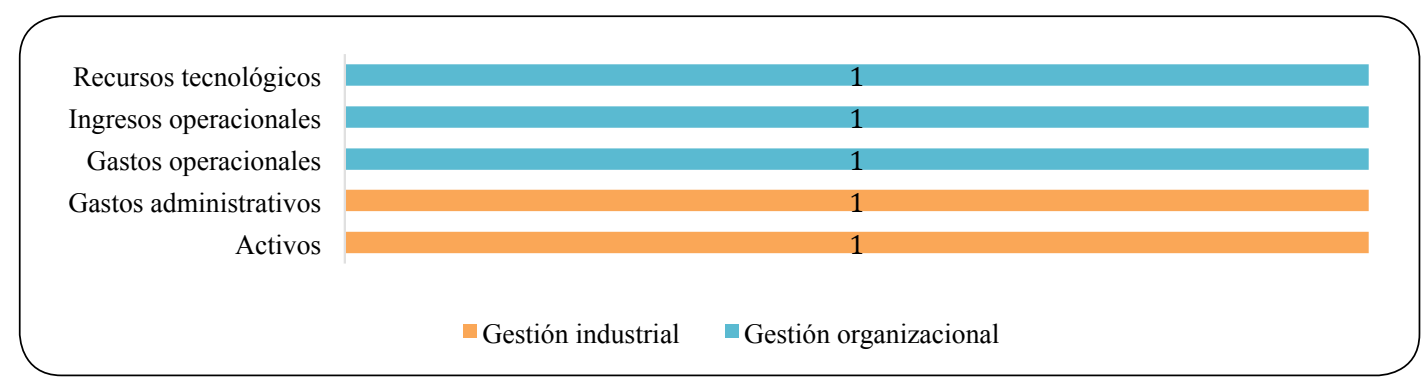

Fuente: elaboración propia. 
Con las salidas (gráfica 25), las ventas y los servicios ofrecidos son las que más coinciden con las empresas transportadoras, donde generan ventajas competitivas prestando servi- cios con calidad y estableciendo estrategias diferenciadoras frente a la competencia (Alfonso, Kalenatic \& Lopez, 2010).

Gráfica 25.

Variables de salida para sección H según los objetivos de gestión industrial y gestión organizacional

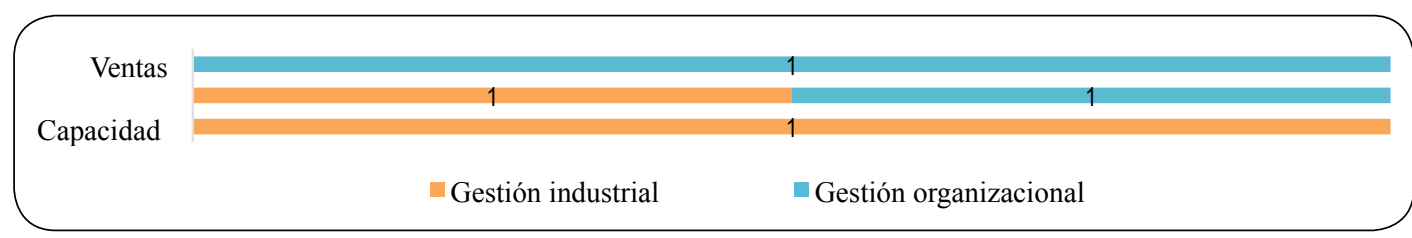

Fuente: elaboración propia.

\section{Sección I-N: Alojamiento y servicios de comida; y actividades de servicios administrativos y de apoyo.}

La sección I y la sección $\mathrm{N}$ coinciden en los estudios en que presentan en común el objetivo de gestión organizacional. Aquí, las empresas relacionadas con el sector hotelero, orientan su gestión en el posicionamiento de su producto en el mercado frente a sus competidores y los precios, hasta la forma que el destino es percibido por el público en general; para ofrecer servicios con mayores comodidades a los clientes y de esta manera aumentar su productividad (Quintero, 2004).

Con respecto a las variables de entrada (gráfica 26 y 27), estas incluyen los recursos materiales, recursos humanos y activos, lo que permite en parte mejorar la productividad de las empresas relacionadas con esta sección, especialmente los hoteles y agencias de viaje. Por lo tanto, al considerar estas empresas que son de servicios el nivel de satisfacción de sus clientes también incide de manera directa en la variable de productividad (Quintero, 2004).

Gráfica 26.

Variables de entrada para sección I según el objetivo de gestión organizacional

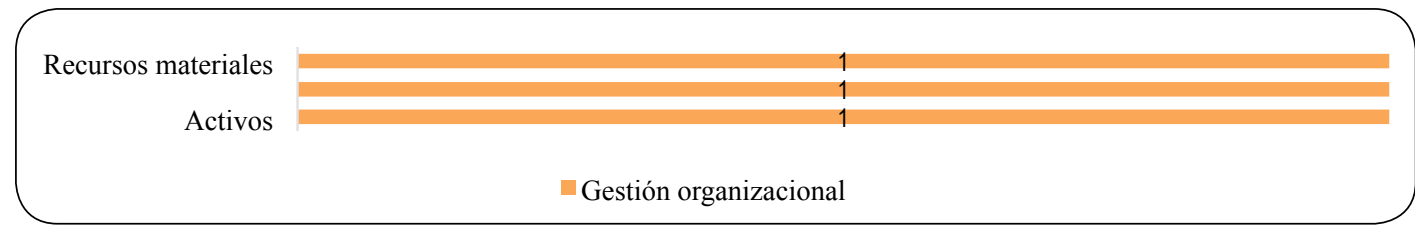

Fuente: elaboración propia.

Gráfica 27.

Variables de entrada para sección I-N según el objetivo de gestión organizacional

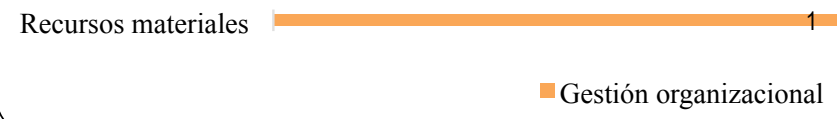

Fuente: elaboración propia. 
Ahora, las salidas (gráfica 28 y 29), tiene también una coincidencia en el objetivo que corresponde a la gestión organizacional. Este es considerado tanto en la administración de los recursos como en las ventas y los ingresos que generan (Cavadia \& Maldonado, 2009).

\section{Gráfica 28.}

Variables de salida para sección I según el objetivo de gestión organizacional

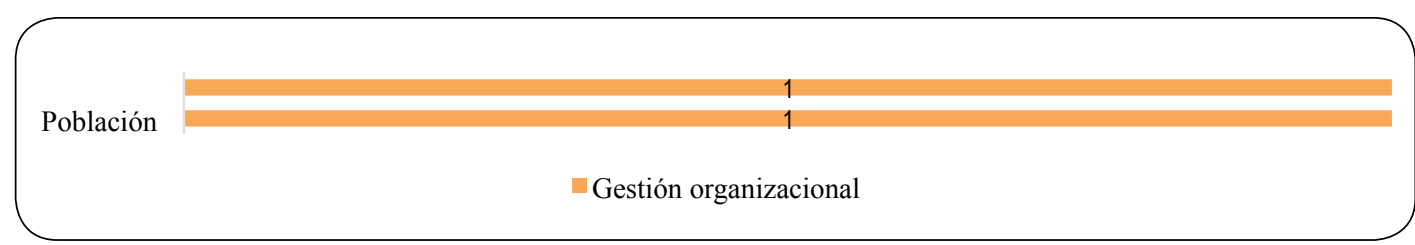

Fuente: elaboración propia.

Gráfica 29.

Variables de salida para sección I-N según el objetivo de gestión organizacional

Fuente: elaboración propia.

\section{Sección J: Información y comu- nicaciones.}

Los objetivos ligados con esta sección son los de gestión industrial y planeación estratégica, que están presentes en cuatro estudios. Las empresas asociadas a esta sección, como las de telecomunicaciones, centran su gestión en lograr la eficiencia económica, partiendo de un uso adecuado de sus recursos, capacidad instalada, el capital y la fuerza laboral (Londoño \& Giraldo, 2009).

En cuanto a las variables de entrada (gráfica 30), recursos tecnológicos y activos influyen en la eficiencia de este tipo de empresas. Por lo que permite concluir que orientan su eficiencia hacia determinadas variables, como son el uso de redes, los activos y otras; hacia la variable personal; reflejando la preocupación por cumplir con la carga laboral y pensional (Gutiérrez, 2007).

\section{Gráfica 30.}

Variables de entrada para sección J según los objetivos de gestión industrial, gestión organizacional, planeación estratégica y producción

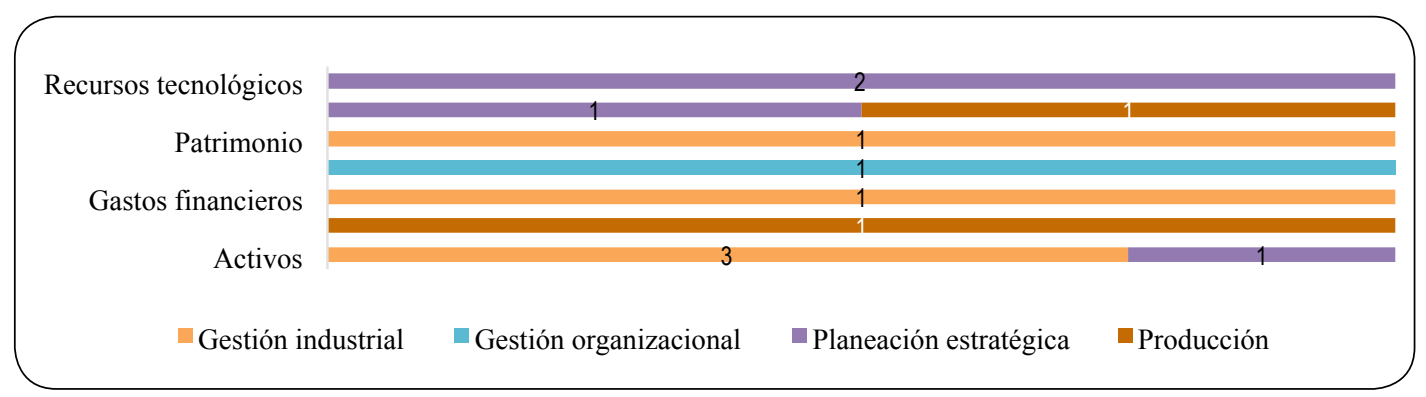

Fuente: elaboración propia. 
Luego, en las salidas (gráfica 31), los objetivos de gestión organizacional e industrial son los más relacionados. Esta relación, la encontramos en empresas de software y tecnologías informáticas vinculadas a esta sección, donde el objetivo fundamental del análisis de la eficiencia operativa a partir de una optimización productiva de sus recursos que emplean, es el de orientar políticas internas y procesos de toma de decisiones, para implementar un ade- cuado control de gestión y mejorar la competitividad en el mercado local y mundial (Mosquera \& Millán, 2013).

También las empresas de información y comunicaciones, tienden a medir su gestión a través de la productividad laboral, donde el capital humano es primordial, y así la necesidad de mantener personal calificado y actualizado (Rodríguez \& Gómez, 2011).

\section{Gráfica 31.}

Variables de salida para sección J según los objetivos de gestión industrial, gestión organizacional, planeación estratégica y producción

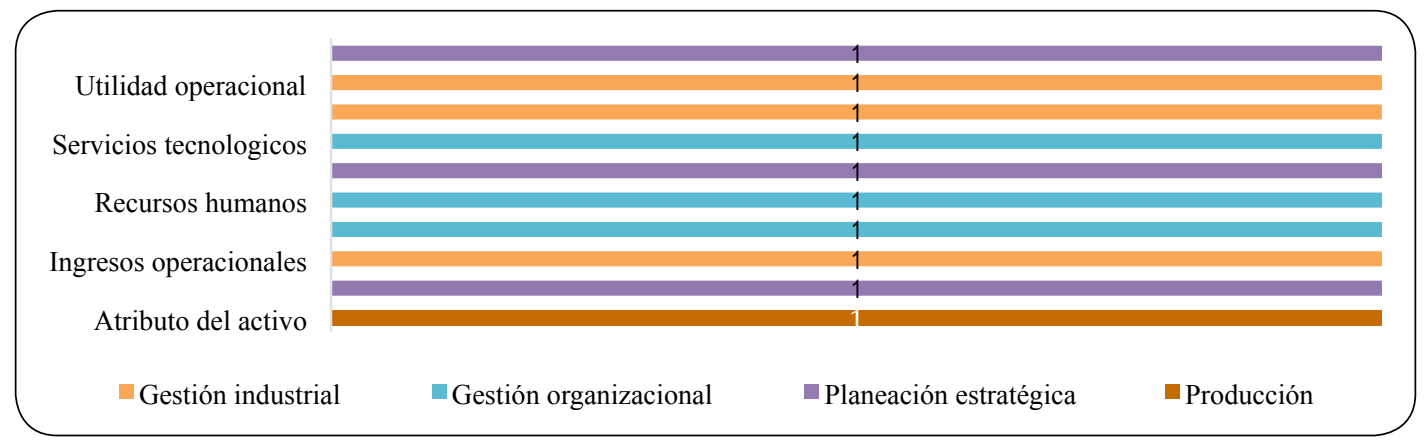

Fuente: elaboración propia.

\section{Sección K: Actividades financie- ras y de seguros.}

El objetivo de manejo de recursos financieros es el más relacionado en esta sección, enfocándose en siete estudios. Con los planteamientos de Fiallos (2003), donde los bancos deben hacer un uso razonable de sus insumos (gastos administrativos, gastos de personal, activos fijos netos y capital) para poder ser eficientes y competitivos en el mercado. El manejo de los recursos en estas organizaciones, está relacionado con la eficiencia en beneficios y la eficiencia en costos, entendiéndose la primera como la capacidad que tiene la firma bancaria para generar utilidades, y la segunda, mide la capacidad que tienen las firmas bancarias para generar su producto al mínimo costo posible; y es precisamente, donde las instituciones bancarias presentan problemas para controlar sus costos reflejando condiciones de competencia ineficientes (Badel, 2002).

Ahora, la ineficiencia entre instituciones financieras ha llevado a los bancos a iniciar un proceso de aprendizaje de mejores prácticas de negocio, con aquellas otras que hacen un uso adecuado de los mismos (Rodríguez, 1998). Siendo así, los bancos pueden ser más competitivos a nivel local e internacional, si reconocen oportunidades nuevas y las aprovechan mediante modelos efectivos de toma de decisiones; así como aprovechando las ventajas de integración con el fin de ampliar el portafolio y lograr la innovación permanente de sus productos y servicios (Berrío \& Muñoz, 2005).

En esta revisión, se tiene que dieciséis investigaciones trabajaron como objetivo el manejo de recursos financieros y la variable de entrada en esta sección CIIU (gráfica 32), más 
asociada con los gastos administrativos. Esta relación, la explica Martínez \& Estrada (2009), afirmando que las compañías de seguros, para incrementar la eficiencia mejoran la eficiencia de asignación. Es decir, la forma como escogen la combinación de sus insumos de producción.

\section{Gráfica 32.}

Variables de entrada para sección K según los objetivos de gestión organizacional $y$ manejo de recursos financieros

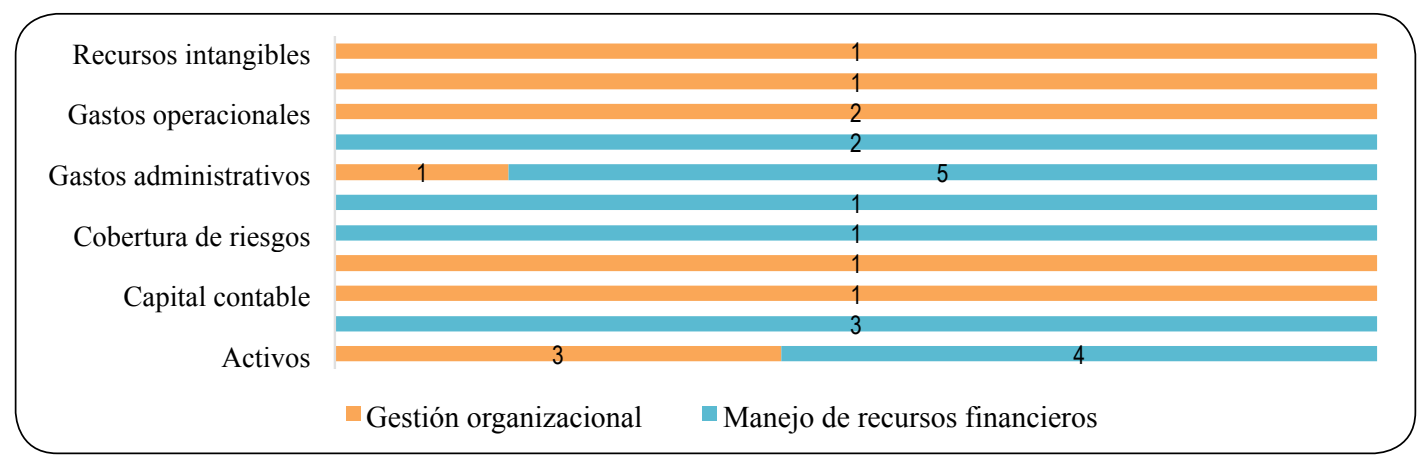

Fuente: elaboración propia.

En cuanto a las salidas (gráfica 33), se tiene que las variables: riesgos por eventos, precios de factores de producción, pérdidas y activos, están relacionados con el objetivo de gestión organizacional. Esta relación, con las compañías de seguros, permite evaluar de una forma cuantitativa los riegos del negocio, debido a la gran competitividad que posibilita permanecer con una posición ventajosa en el mercado (Londoño, 2004).

\section{Gráfica 33.}

Variables de salida para sección K según los objetivos de gestión organizacional y manejo de recursos financieros

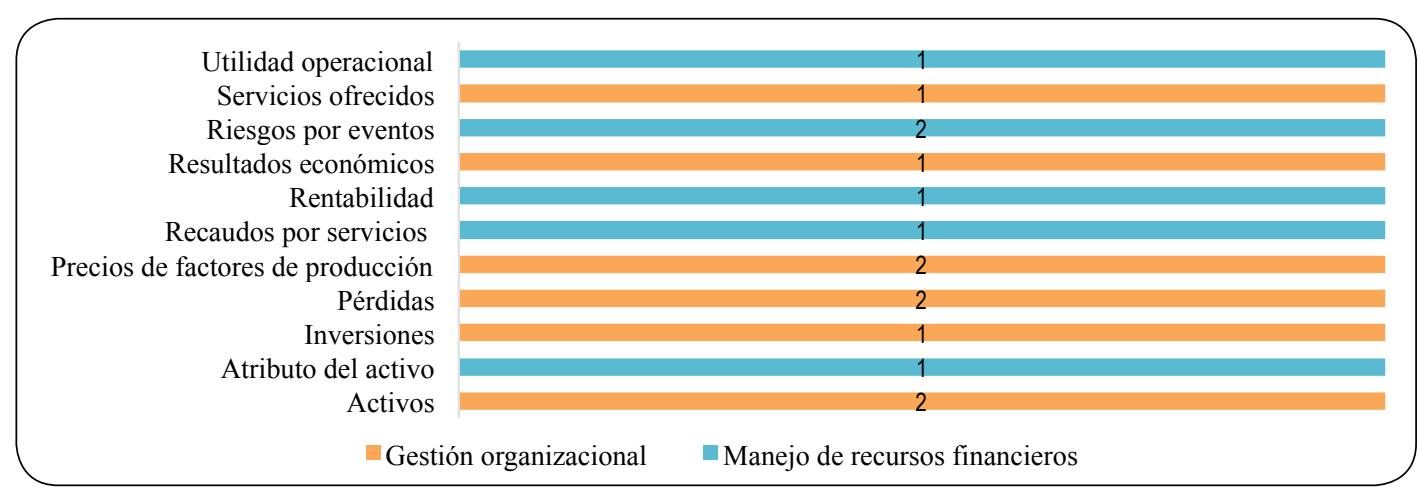

Fuente: elaboración propia.

Sección O: Administración pública y defensa; planes de seguridad social de afiliación obligatoria.

La gestión pública es el objetivo más relacionado con esta sección presente en trece investigaciones. Donde, cinco están enfocadas al desempeño fiscal en departamentos y municipios del territorio nacional, buscando analizar la eficiencia con que invierten los recursos asignados para ciertos rubros específicos, como las transferencias para salud, educación y sanea- 
miento básico (Pérez, 2005). En este sentido, la inversión de recursos representada en las transferencias, tienen un papel preponderante en la eficiencia de la gestión pública, para medir los niveles de eficiencia necesarios cuando se contrastan los insumos con los productos (León \& Ramírez, 2007).

En general, el tema de manejo de recursos en entidades públicas específicamente como entidades territoriales, cobra su importancia por la financiación de la inversión municipal y departamental y por la magnitud que esta representa en el gasto público consolidado del país (Porras, 2005). Respecto a esto la ley 152 de julio de 1994:

"Por la cual se establece la ley Orgánica del Plan de Desarrollo", en su capítulo X "Procedimientos para los planes territoriales de desarrollo", se establece en su artículo 42 "Evaluación. Corresponde a los organismos departamentales de planeación efectuar la evaluación de gestión y resultados de los planes y programas de desarrollo e inversión tanto del respectivo departamento, como de los municipios de su jurisdicción (Chediak, 2008, p. 20).

Dos estudios se centraron en el tema del manejo de las regalías petroleras, donde se evalúa la relación entre las regalías directas y las coberturas mínimas alcanzadas por los municipios productores de petróleo, para medir la eficiencia en la administración gubernamental (Armenta, Alberto, \& Prieto, 2012; Díaz, 2008).

En cuanto a las variables de entrada (gráfica 34), los estudios manifestaron cómo los recursos intangibles y los servicios ofrecidos son participes del desempeño del sector público, donde la eficiencia de las entidades estatales, como son el caso de las CAR (Corporaciones Autónomas Regionales), se mide mediante los proyectos ejecutados y las personas atendidas con base en los servicios que prestan; teniendo especial cuidado en la calidad de los resultados para garantizar transparencia (Britto, Buitrago, Puerto \& Ramírez, 2013).

\section{Gráfica 34.}

Variables de entrada para sección $O$ según los objetivos de gestión organizacional, gestión pública, manejo de recursos financieros y manejo de recursos naturales

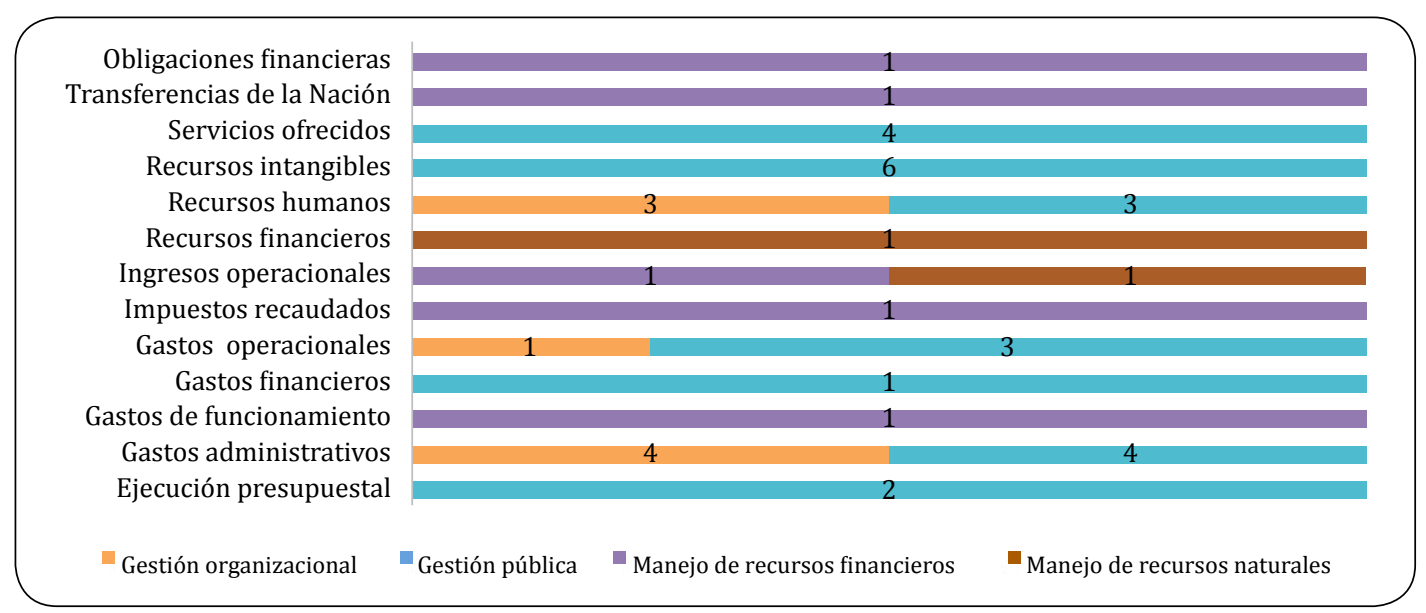

Fuente: elaboración propia.

En las salidas (gráfica 35), las variables: resultado económico y resultado del servicio, están relacionados con el objetivo de gestión pública. Por esta relación, las entidades públicas como las AFP (Administradora de Fondos de Pensiones), basan la toma de decisiones en 
la generación de ingresos e inversión como producto de su función de administración de los fondos, y en la obtención de rentabilidades para los trabajadores (Sánchez, 2011).

\section{Gráfica 35.}

Variables de salida para sección $O$ según los objetivos de gestión organizacional, gestión pública, manejo de recursos financieros y manejo de recursos naturales

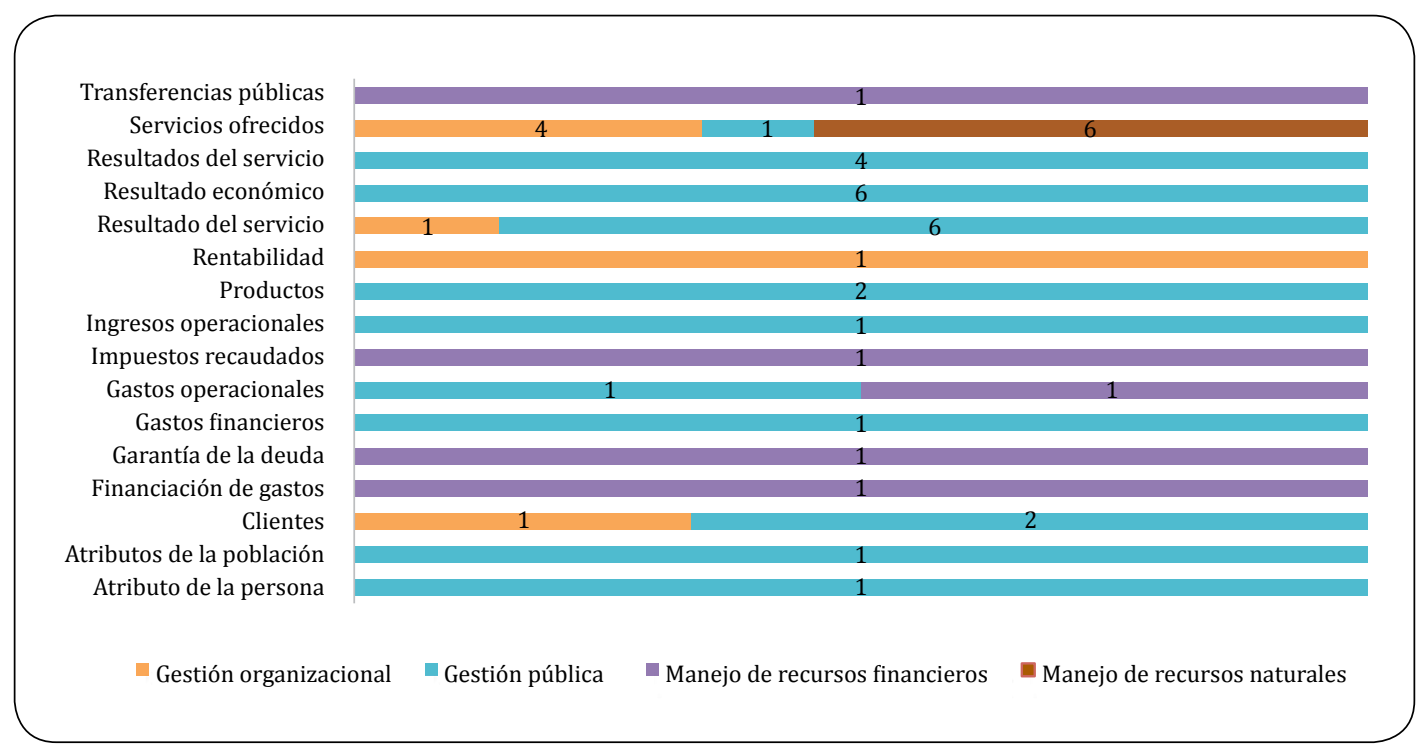

Fuente: elaboración propia.

\section{Sección Q: Actividades de aten- ción de la salud humana y de asistencia social.}

El objetivo gestión pública es el más relacionado en esta sección, enfocándose en seis estudios. Según Nupia y Sánchez (2001), la gestión de las instituciones públicas como los hospitales, se mide de acuerdo con la estructura de ingresos y gastos, la demanda de servicios y la localización geográfica. Por eso, la eficiencia de las instituciones de salud está determinada por la medición de productividad, para establecer si los hospitales hacen un manejo óptimo de sus recursos para sobrevivir en un mercado altamente competitivo (Hincapié \& Salazar, 2009).

Ahora, teniendo en cuenta la anterior caracterización, el nivel de servicio en los hospitales se mide por su nivel de comple- jidad, determinado por los servicios que ofrece, siendo los de nivel I, II y III, de baja, mediana y alta complejidad respectivamente (Toro \& Mutis, 2006), donde no existe restricciones en la prestación de servicios, y la complejidad está dada por su tecnología y por el cuerpo asistencial más que por los servicios que presta. Entonces, los hospitales públicos para tener mejores prácticas, requieren trabajar con variables que expresen la complejidad de los pacientes atendidos (días de estancia), tamaño del hospital (número de camas) y nivel tecnológico del hospital (gastos de funcionamiento) (Rodríguez \& Suarez, 2011).

Luego, se tiene que las variables: servicios ofrecidos y recursos intangibles (gráfica 36), están muy relacionadas con el desempeño de las entidades de salud en el sector público. Para Aristizabal (2004), el desempeño de los hospitales públicos está determinado por los recursos utilizados y los resultados generados. 


\section{Gráfica 36. \\ Variables de entrada para sección Q según los objetivos de gestión organizacional, gestión pública y planeación estratégica}

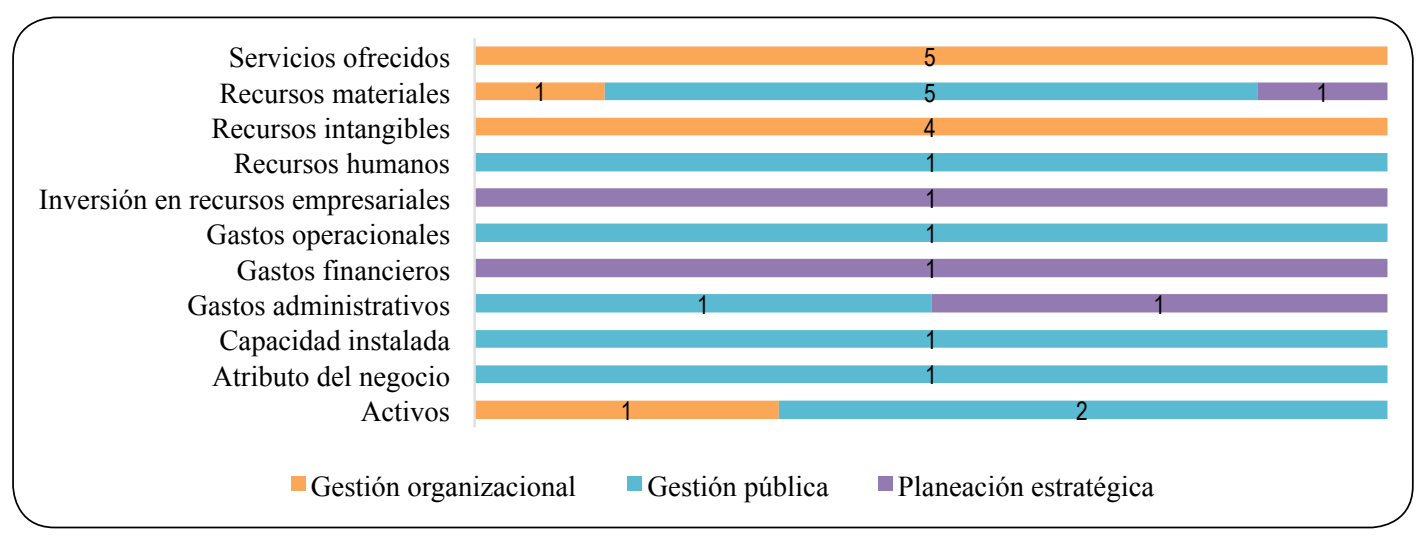

Fuente: elaboración propia.

En las salidas (gráfica 37), la variable servicios ofrecidos es la más relacionada con el objetivo de gestión pública. Esto se debe, a que las entidades públicas relacionadas con el sector salud, están obligadas a trabajar con altos niveles de productividad y calidad en la prestación de servicios, pues estos son los principales determinantes en la capacidad de supervivencia (Navarro, Maza, \& Rafael, 2009).

\section{Gráfica 37.}

Variables de salida para sección Q según los objetivos de gestión organizacional, gestión pública y planeación estratégica

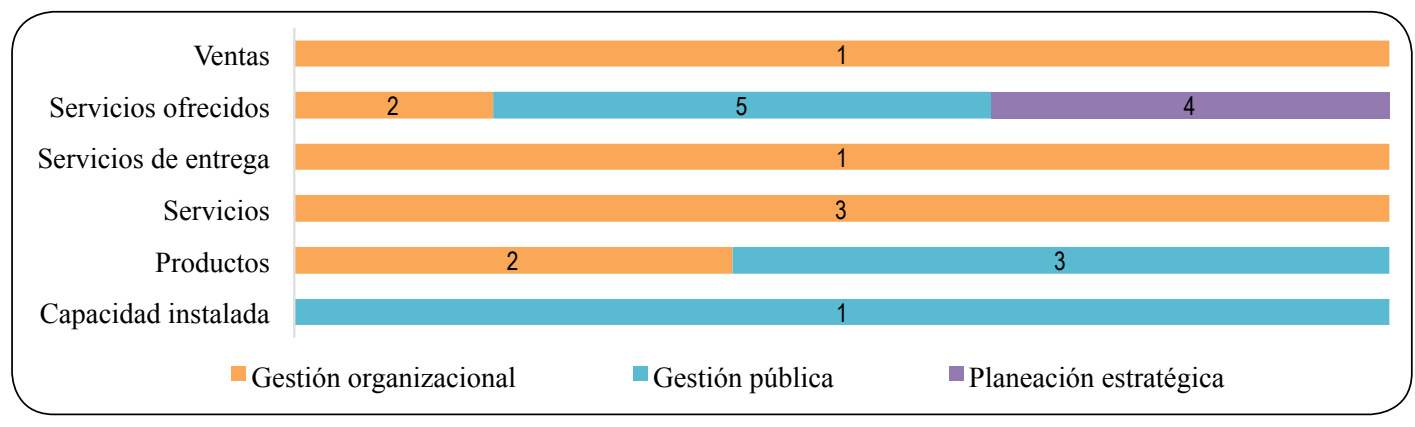

Fuente: elaboración propia.

\section{Conclusiones e implicaciones}

Según la tendencia de la implementación de la metodología DEA para medir eficiencias técnicas relativas en las organizaciones, su uso se incrementó en un $300 \%$ del año 2002 al año 2003, a partir de este año la tendencia se mantiene hasta el año 2013 que es prácticamente el lapso de tiempo analizado.
Esto nos permite inferir, que la metodología es actualmente usada por las organizaciones para medir sus eficiencias técnicas relativas y de esta manera mejorar sus procesos para ser más productivas.

Con respecto a la frecuencia de la implementación de la metodología DEA en las actividades económicas del CIIU, se tiene que las ac- 
tividades de administración pública y defensa, y planes de seguridad la implementaron en un 22 $\%$; las actividades financieras y de seguros en un $12 \%$; las actividades de agricultura, ganadería, caza, silvicultura y pesca en un $12 \%$; las actividades de suministro de electricidad, gas, vapor y aire en un $11 \%$ y las actividades de la salud humana y de asistencia social en un $11 \%$. De esta manera, estas 5 actividades económicas del CIIU corresponde al $42 \%$ de las actividades CIIU clasificadas por el DANE y cuentan con el $70 \%$ de las investigaciones implementando la metodología DEA.

En cuanto a los objetivos de medición de eficiencia determinados en la investigación, se tiene que el $50 \%$ de estos fueron contemplados en el $74 \%$ de las investigaciones. Siendo así, el objetivo de la gestión organizacional el más representativo con el $29 \%$ de las investigaciones; el objetivo de gestión pública con el $17 \%$; el objetivo de gestión industrial con el $16 \%$ y por último, el objetivo de manejo de recursos financieros con el $12 \%$. De esta manera, se encuentra una relación lógica entre las actividades de administración pública y defensa, y planes de seguridad; con el objetivo de gestión organizacional.

Por último, y para tener en cuenta en futuras revisiones bibliográficas asociadas a investigaciones con la aplicación de la metodología DEA, se evidenció que los resultados principalmente se plasman en los artículos científicos en un $55 \%$ y trabajos de grado en un $35 \%$.

\section{Nota}

${ }^{1}$ La codificación CIIU (Clasificación Industrial Internacional Uniforme) fue elaborada por la Organización de las Naciones Unidas y adaptado para Colombia por el DANE. Es utilizada con el propósito de clasificar las actividades económicas de los empresarios colombianos de una manera clara y precisa, y se empezó a implementar a partir del año 2000.

\section{Referencias}

Alfonso, E., \& Gutiérrez, E. (2007). Evaluación de eficiencia radial en el sector agroindustrial a través del modelo DEA VRS con salidas no deseada. Revista Científica y Tecnológica de la Facultad de Ingeniería, 12(2), 39-45.

Alfonso, E., Kalenatic, D., \& Lopez, C. (2010). Modeling the synergy level in a vertical collaborative supply chain through the IMP interaction model and DEA framework. Bogotá: Universidad de la Sabana.

Aristizabal, G., \& Salazar, R. (2011). Eficiencia financiera del sector industrial de agroquímicos, Colombia, 2005. Economía, Gestión y Desarrollo, 11, 91-119.

Aristizabal, J. A. (2004). Aplicación de análisis envolvente de datos a hospitales públicos de segundo y tercer nivel. Bogotá: Universidad de los Andes.

Armenta, R., Alberto, B., \& Prieto, W. (2012). Medición de la eficiencia en el uso de las regalías petroleras: una aplicación del análisis envolvente de datos. Finanzas y Politica Económica, 1(4), 13-32.

Badel, A. (2002). Sistema Bancario Colombiano: ¿Somos eficientes a nivel internacional?. Bogotá: Departamento Nacional de Planeación.

Berrío, D., \& Muñoz, A. (2005). Análisis de la eficiencia relativa del sistema bancario en Colombia en el periodo 1993-2003 y propuesta estratégica de fortalecimiento. Pensamiento y Gestión, 18, 1-36.

Britto, R., Buitrago, O., Puerto, J., \& Ramírez, J. (2013). Relative Efficiency in Government Environmental Entities: An Evaluation of the Regional Autonomous Corporations 
(CAR) of Colombia. Ingeniería y Universidad. Ing. Univ., 17(1), 205-223.

Cámara de Comercio de Bogotá -[CCB]. (2012). Todo sobre el código CIIU. Recuperado de http://www.ccb.org.co/Inscripciones-y-renovaciones/Todo-sobreel-codigo-CIIU

Cano, L. (2007). Descentralización fiscal y eficiencia en los servicios sociales a nivel territorial en Colombia. Equidad y Desarrollo, 8, 7-24.

Cavadia, R., \& Maldonado, M. (2009). Análisis de la eficiencia del sector hotelero del distrito turístico de Cartagena por medio del DEA (Análisis envolvente de datos). (Tesis de pregrado). Universidad de Cartagena, Colombia.

Cayon, E. (2001). Measuring relative efficiencies in the shoe industry sector in Colombia: a DEA approach. Bogotá: Pontificia Universidad Javeriana.

Cegarra, J. (2004). Metodología de la Investigación Cientifica y Tecnológica. Madrid: Ediciones Díaz de Santos.

Cervera, A., Oviedo, W., \& Pineda, J. (2013). Revisión bibliográfica de la aplicación de la metodología DEA en el ámbito educativo colombiano. Revista Civilizar Ciencias Sociales y Humanas, 13(25), 133-156.

Chediak, F. (2008). La técnica DEA para medir y analizar la eficiencia de municipios del departamento del Tolima, año 2006. Pereira: Universidad Tecnológica de Pereira.

Comisión de Regulación de Agua Potable y Saneamiento Básico -[CRA]. (2005). Determinación de datos base para la estimación de modelos de eficiencia comparativa DEA. Bogotá. Recuperado de http://tramitesccu.cra.gov.co/normatividad /admon1202/files/Documento_de_trabajo_Res_312.pdf

Comisión de Regulación de Energía y Gas [CREG]. (2000). Metodología para la fijación de los gastos de AO\&M de las empresas transportadoras de gas en Colombia. Bogotá. Recuperado de http:// http://apolo.creg.gov.co/Publicac.nsf/1c 09d18d2d5ffb5b05256eee00709c02/0d a178fb881da9fc0525785a007a6563/\$FI LE/D-134-AOM\%20TRANSPORTE.pdf

Cunha, R., \& Garzón, F. (2007). Performance based potable water and sewer service regulation the regulatory model. Cuadernos de administración, 20(34), 283-298.

Departamento Administrativo de Ciencia, Tecnología e Innovación -[Colciencias]. (2010). Documento guía: Servicio permanente de Indexación de Revistas de Ciencia, Tecnología e Innovación Colombianas. Recuperado de http://scienti. colciencias.gov.co:8084/publindex/docs/ informacionCompleta.pdf

Díaz, J. (2008). Eficiencia en el uso de regalías petroleras en Aguazul Casanare. Bogotá: Universidad de la Sabana.

Duncan, G. (2008). El uso del DEA para la estimación del factor $\mathrm{X}$ en la definición de tarifas portuarias. Colombia Internacional, 68, 44-67.

Fiallos, J. (2003). Metodología de la evaluación relativa para cooperativas de aporte $y$ crédito. Recuperado de http://dspace. uniandes.edu.co/xmlui/bitstream/handle/1992/243/mi_1065.pdf?sequence=1

Gamarra, J. (2004). Eficiencia Técnica Relativa de la Ganadería doble propósito en la Costa Caribe. Cartagena: Banco de la República. 
García, A. (2005). Metodología para la remuneración de costos eficientes de administración, operación y mantenimiento de empresas de transmisión usando análisis envolvente de datos. Bogotá: Universidad de los Andes.

González, R. (2010). Utilización del análisis envolvente de datos (DEA) en el desarrollo de una metodología para el establecimiento de costos eficientes de remuneración, en la administración, operación y mantenimiento de los sistemas de distribución eléctrica. Bogotá: Universidad Nacional de Colombia.

Guio, O., \& Monroy, I. (2003). Medición de la eficiencia relativa de agentes generadores de energía eléctrica en Colombia (Tesis de pregrado). Pontificia Universidad Javeriana, Bogotá, Colombia.

Gutiérrez, J. (2007). Análisis del sector de la telefonía local en Colombia mediante técnicas estadísticas multivariadas $y$ Análisis Envolvente de Datos durante el periodo 1997-2001. Bogotá: Universidad de los Andes.

Hincapié, M., \& Salazar, P (2009). Medición de la eficiencia técnica relativa de los hospitales de Risaralda. Pereira: Universidad Católica Popular de Risaralda.

León, R., \& Ramírez, L. (2007). Descentralización fiscal y eficiencia en los servicios sociales a nivel territorial en Colombia. Equidad y Desarrollo, 8, 7-24.

Ley 142 de 1994. Por la cual se establece el régimen de los servicios públicos domiciliarios y se dictan otras disposiciones. Diario Oficial No. 41.433. Congreso de la República de Colombia, julio de 1994.

Ley 143 de 1994. Por la cual se establece el régimen para la generación, interconexión, trasmisión, distribución y comercialización de electricidad en el territorio nacional, se conceden unas autorizaciones y se dictan otras disposiciones en materia energética. Diario Oficial No. 41.434. Congreso de la República de Colombia, julio de 1994.

Ley 152 de 1994. Por la cual se establece la Ley Orgánica del Plan de Desarrollo. Diario Oficial No. 41.450. Congreso de la República de Colombia, julio de 1994.

Londoño, L. (2004). Análisis y determinación de eficiencia relativa de clientes asociados al negocio de seguros de transporte (Tesis de pregrado). Universidad de La Sabana, Bogotá, Colombia.

Londoño, L., \& Giraldo, Y. (2009). Análisis Envolvente de Datos -DEA-: Una aplicación al sector de telecomunicaciones de países de medianos ingresos. Ecos de Economía, 13(28), 53-73.

López, J., \& Fernández, S. (2009). Aplicación de la técnica de la (Data Envelopment Analysis) en la determinación de eficiencia de centros de costos de producción. Scientia et Technica, 37, 395-400.

Luna, R. (2012). Determinantes de la eficiencia técnica en la agroindustria de aceite de palma en el departamento del Magdalena. Ingeniería Solidaria, 8(14), 8-18.

Martínez, J., \& Estrada, D, (2009). Análisis de eficiencia y productividad en la industria de seguros colombiana. Fasecolda. Recuperado de http://www.fasecolda. com/files/6113/9101/5707/analisis_de eficiencia_y_productividad_en_la_industria_de_seguros_colombiana.pdf

Maza, F., Navarro, J., \& Puello, J. (2012). ¿Fue eficiente la asignación de recursos en el suministro de agua potable en el 
Departamento de Bolívar - Colombia en el periodo 2007-2008?. Entramado, 8(1), 58-70.

Montoya, O., \& Soto, J. (2010). Estimación de la eficiencia técnica de las economías de los departamentos cafeteros de Colombia, por el método de programación lineal análisis envolvente de datos (DEA). Scientia et Technica, 1(44), 348-353.

Mosquera, H., \& Millán, J. (2013). Competitividad en empresas innovadoras de software y tecnologías informáticas. Cuadernos de administración, 29(49), 37 - 44.

Navarro, J., Maza, F., \& Rafael, V. (2009). La eficiencia de los hospitales colombianos en el contexto Latinoamericano. Una aplicación de Análisis Envolvente de Datos (DEA) en un grupo de hospitales de alta complejidad, 2009. Ecos de Economía, 15(33), 71-93.

Nupia, O., \& Sánchez, F. (2001). Eficiencia de los hospitales públicos de Bogotá. Desarrollo y sociedad, 48, 101-136.

Oviedo, W., \& Rodríguez, G. (2011). Medición de la eficiencia técnica relativa de las fincas asociadas a Coounión en Guasca Cundinamarca. Revista MVZ Córdoba, 16(2), 2616-2627.

Pardo, C., \& Cotte, A. (2011). La eficiencia energética en la industria manufacturera colombiana: una estimación con Análisis Envolvente de Datos-DEA y Datos de Panel. Economía, Gestión y Desarrollo, $11,39-58$.

Parra, D., \& Toro, I. (2006). Método y conocimiento. Metodología de la investigación: Investigación cualitativa/investigación cuantitativa. Medellín: Universidad EAFIT.
Perdomo, J., \& Mendieta, J. (2007). Factores que afectan la eficiencia técnica y asignativa en el sector cafetero colombiano: una aplicación con análisis envolvente de datos. Desarrollo y Sociedad, 1(60), 1-45.

Pérez, J. (2005). Evaluación de la eficiencia de los departamentos en Colombia mediante análisis envolvente de datos - DEA. Bogotá: Universidad de los Andes.

Porras, O. (2005). Metodología para la medición y análisis del desempeño municipal. Bogotá: Departamento Nacional de Planeación.

Porter, M. (2006). Estrategia y ventaja competitiva. Barcelona: Ediciones Deusto.

Quintero, J. (2004). Eficiencia técnica y cambio en la productividad en las empresas turísticas de Cartagena, 2001-2004: ¿Qué tan competitivos son los hoteles $y$ las agencias de viajes locales? (Serie de estudios sobre la competitividad de Cartagena No. 10). Recuperado de http:// www.cccartagena.org.co/sites/default/ files/2009062553_inv_no10ef-1.pdf

Rodríguez, G. (1998). Aproximación preliminar al estudio de la eficiencia de 5 subsectores de la economía colombiana usando DEA. Innovar 11, 65-97.

Rodríguez, G. (2003a). Medición de la eficiencia relativa en tres subsectores de la economía colombiana desde 1993 a 1999 utilizando Data Envelopment Analysis (D.E.A.). Innovar, 13(21), 145-182.

Rodríguez, G. (2003b). Medición de la eficiencia relativa en dos subsectores de la economía colombiana desde 1993 a 2002 utilizando DEA. Innovar 13(22), 121-144.

Rodríguez, G. (2011). Indicadores DEA de eficiencia y productividad. Bogotá: Universidad Nacional de Colombia. 
Rodríguez, G., \& Suarez, D. (2011). Evaluación de la eficiencia de las (entidades promotoras de salud - EPS) públicas y privadas a través de un modelo multivariado de análisis envolvente de datos (DEA). Bogotá: Universidad de La Salle.

Rodríguez, J. (2004). Aplicación de Análisis Envolvente de Datos a la Evaluación de Eficiencia en Gastos Administrativos para la Industria de Seguros Generales en Colombia. Bogotá: Universidad de los Andes.

Rodríguez, J. L., \& Gómez, D. (2011). Crecimiento y eficiencia de la industria editorial de Bogotá, Colombia. CENES, 31(53), 201-224.

Rojas, L. (2011). Aplicación del análisis envolvente de datos para la priorización del mantenimiento en circuitos de distribución de energía eléctrica de Media Tensión. CIER, 59, 47-53.

Sánchez, M. (2011). Sociedades Administradoras de Pensiones y Grupos Económicos. Efectos de la afiliación sobre el desempe- ño de las AFP en el periodo 2001-2010. Bogotá: Universidad Nacional de Colombia.

Sepúlveda, M. (2014). Análisis de eficiencia técnica y estudio de casos en los cultivos de flores de la Sabana de Bogotá (Colombia). Pensamiento y Gestión, 36, 289-323.

Tolosa, B. (2013). Evaluación de eficiencia en el sector de distribución de energía eléctrica en Colombia empleando la metodología de análisis envolvente de datos - DEA (Tesis de maestría). Universidad Nacional de Colombia, Bogotá.

Toro, E., \& Mutis, H. (2006). Medición de la eficiencia de las instituciones prestadoras de servicios de salud pública mediante técnicas multivariadas y análisis envolvente de datos. Bogotá: Universidad de los Andes.

Urrego, D. (2010). Eficiencia de la industria del agua potable en Colombia: Una aproximación a partir del Análisis Envolvente de Datos con factores ambientales. Barcelona: Universidad Autónoma de Barcelona. 